\title{
WHAT IS THE CORRECT LOGIC OF NECESSITY, ACTUALITY AND APRIORITY?
}

\author{
PETER FRITZ \\ University of Oxford
}

\begin{abstract}
This paper is concerned with a propositional modal logic with operators for necessity, actuality and apriority. The logic is characterized by a class of relational structures defined according to ideas of epistemic two-dimensional semantics, and can therefore be seen as formalizing the relations between necessity, actuality and apriority according to epistemic two-dimensional semantics. We can ask whether this logic is correct, in the sense that its theorems are all and only the informally valid formulas. This paper gives outlines of two arguments that jointly show that this is the case. The first is intended to show that the logic is informally sound, in the sense that all of its theorems are informally valid. The second is intended to show that it is informally complete, in the sense that all informal validities are among its theorems. In order to give these arguments, a number of independently interesting results concerning the logic are proven. In particular, the soundness and completeness of two proof systems with respect to the semantics is proven (Theorems 2.11 and 2.15), as well as a normal form theorem (Theorem 3.2), an elimination theorem for the actuality operator (Corollary 3.6), and the decidability of the logic (Corollary 3.7). It turns out that the logic invalidates a plausible principle concerning the interaction of apriority and necessity; consequently, a variant semantics is briefly explored on which this principle is valid. The paper concludes by assessing the implications of these results for epistemic two-dimensional semantics.
\end{abstract}

§1. Introduction. Epistemic two-dimensional semantics as proposed by David Chalmers, e.g., in Chalmers (2004), provides an account of meaning that allows a possible world semantics of necessity as well as apriority. The notions of necessity and apriority intended here are those distinguished by Kripke (1972); the first is sometimes called metaphysical necessity. They can roughly be paraphrased by saying that necessary is what could not have failed to be the case, and a priori is what can be known in an a priori way. In Fritz (2013), a propositional modal logic with operators for necessity, actuality and apriority is defined which captures the relevant ideas of epistemic two-dimensional semantics. In particular, a class of relational structures is defined, and it is argued that it represents the evaluation of sentences according to epistemic two-dimensional semantics, and that therefore, the logic characterized by this class captures the relations of the three modalities according to epistemic two-dimensional semantics. Epistemic two-dimensional semantics is a controversial theory, and philosophers who do not want to commit themselves to it can't justify the correctness of this logic using its semantics. This raises the question whether there is a way of arguing for its correctness that is not based on epistemic two-dimensional semantics. The main aim of this paper is to outline such an argument.

Received: September 9, 2012 
In this introductory section, I first summarize the definition of the logic in Fritz (2013). I then consider what it takes for this to be the correct logic of necessity, actuality and apriority, and sketch the overall strategy of arguing for the correctness of the logic which will be outlined in subsequent sections. As I will only be concerned with epistemic twodimensional semantics, rather than other variants of two-dimensional semantics, I will drop the qualifier epistemic from now on for brevity.

1.1. A Logic for Two-Dimensional Semantics. Syntactically, the logic discussed in Fritz (2013) uses a standard propositional modal language with three operators for the three modalities. Letters like $p$ and $q$ are used as proposition letters; $\neg$ and $\wedge$ are used as primitive Boolean operators; and $\square$ is used for necessarily, $A$ for a priori, and @ for actually. $\diamond$ and $C$ are used as the duals of $\square$ and $A$. It is assumed that $\diamond$ is used for possibly, and the symbol $C$ is motivated by the suggestion in Chalmers (2004, p. 219) that conceivability and apriority are dual in the required way, although this won't be assumed in the following, and so $C$ shouldn't be assumed to formalize conceivably. Note that in some of the references cited below, the actuality operator is written $A$, the symbol I use for the apriority operator.

The basic idea behind the semantics is that truth is relativized to two indices, a metaphysical possibility and an epistemic possibility. Necessity is interpreted as truth in all metaphysical possibilities, keeping the epistemic possibility fixed. Apriority is interpreted as truth in all epistemic possibilities and corresponding metaphysical possibilities. Actuality is interpreted as truth in the metaphysical possibility corresponding to the epistemic possibility, keeping the epistemic possibility fixed. In fact, the version of the semantics presented in the following definition identifies metaphysical and epistemic possibilities, since this simplification makes no difference to the logic of the three modalities considered here. See Fritz (2013) for more on the philosophical motivation of this model theory and for brief comparisons with similar logics such as the ones found in Davies \& Humberstone (1980) or Restall (2012).

In a first step to specify the formal model theory, a class $\mathrm{M}$ of Kripke frames called matrix frames is defined in Fritz (2013) as the class of frames $\mathfrak{F}=\left\langle W, R_{\square}, R_{@}, R_{A}\right\rangle$, where $W=S \times S$ for some set $S$, and the relations are given by the following conditions:

- $\langle x, y\rangle R_{\square}\left\langle x^{\prime}, y^{\prime}\right\rangle$ iff $y=y^{\prime}$

- $\langle x, y\rangle R_{@}\left\langle x^{\prime}, y^{\prime}\right\rangle$ iff $y=y^{\prime}$ and $x^{\prime}=y^{\prime}$

- $\langle x, y\rangle R_{A}\left\langle x^{\prime}, y^{\prime}\right\rangle$ iff $x^{\prime}=y^{\prime}$

From this, a class of frames with distinguished elements is derived. As defined in Segerberg (1971), a frame with distinguished elements, in short FWDE, is a frame to which a subset of the set of points is added. Models are obtained from these as in the case of frames by adding a valuation function, and the definition of truth at a point in a model stays the same. Only the definitions of validity and consequence are changed by restricting them to the distinguished points. Logics characterized by classes of FWDEs need not be normal, as they need not be closed under the rule of generalization (if $\varphi$ is a theorem so is $\nabla \varphi$ for any modal operator $\nabla$ ). But they are quasi-normal, which means that they contain the smallest normal modal logic $\mathbf{K}$ and are closed under modus ponens and uniform substitution. With this, the formal semantics of the logic for two-dimensional semantics is given by the class MD of matrix FWDEs, which are defined as the FWDEs $\mathcal{F}=\left\langle W, R_{\square}, R_{@}, R_{A}, D\right\rangle$ such that $\left\langle W, R_{\square}, R_{@}, R_{A}\right\rangle$ is a matrix frame and $D=\{\langle x, x\rangle$ : $x \in S\}$, where $S$ is the set such that $W=S \times S$. Note that I distinguish between frames and FWDEs, as well as models based on such structures, using different fonts. E.g., I write 
$\mathfrak{F}$ and $\mathfrak{M}$ for frames and models based on them, and $\mathcal{F}$ and $\mathcal{M}$ for FWDEs and models based on them.

1.2. A Question of Correctness. If you are interested in the logic of necessity, actuality and apriority but are not committed to two-dimensional semantics, you might wonder whether we can argue independently of two-dimensional semantics that the logic characterized by MD is the correct one. But what exactly does it mean for a logic to be the correct logic of necessity, actuality and apriority? Since here, we will take a modal logic to be just a set of formulas, a trivial answer follows from the identity criterion for sets: To be the correct logic of the three modalities is just to contain the same formulas as the correct logic. Let's call the formulas that are in the correct logic informally valid. Then we can split up the claim that the logic characterized by MD is the correct logic into two parts, adapting some familiar terminology: Say that it is informally sound if it contains only formulas that are informally valid, and informally complete if it contains all formulas that are informally valid. To be the correct logic can now be described as being informally sound and complete. Of course, unless we have an independent account of which formulas are informally valid, these are all just slight variations of saying the same thing. Before saying more about informal validity, let me point out that there are reasons for thinking that this account of being the correct logic of the three modalities is incomplete. (For brevity, let me just say that a logic is correct instead of saying that it is the correct logic of necessity, actuality and apriority, or whatever else it formalizes.)

One might argue that the basic concept in logic is not that of a formula being valid, but that of an argument being valid. Then the problem with the above account would be the assumption that the correct logic of the three modalities is given as a set of formulas. If this is right, then it would be more accurate to say that a logic is correct if it counts all and only the informally valid arguments as (formally) valid. Adapting standard terminology, we might call this strong correctness, and the notion of correctness described in the previous paragraph weak correctness. As above, these can then be divided up into strong and weak informal soundness and completeness.

To consider this in a bit more detail, let me assume the standard representation of arguments in modal logics as given by a set of premises and a single conclusion. As we will see later (as an immediate consequence of Theorem 2.11), the consequence relation over MD is compact (i.e., what is a consequence of a set of formulas is a consequence of a finite subset). So it follows from natural assumptions about informal validity that the logic characterized by MD is strongly informally sound if it is weakly informally sound. (The assumptions are an informal analog of the deduction theorem and the claim that a formula is informally valid if it follows informally from no premises.) But to infer that the logic is strongly informally complete from the assumption that it is weakly informally complete, we need the claim that informal consequence is compact, which we might call informal compactness. That it holds in the current modal context is by no means obvious, as pointed out in Cresswell (2009, p. 63). So what I say below about informal completeness should be thought of as only pertaining to weak informal completeness, and leaving the question of strong informal completeness open, or depending on the assumption of informal compactness. While I acknowledge these complications, I won't consider them in the following, and therefore also return to the original terminology; i.e., writing just informal soundness and informal completeness for what has here been called weak informal soundness and completeness.

Taking a stand on the correct understanding of informal validity is beyond the scope of this paper. But to see which parts of the arguments for informal soundness and 
completeness that will be outlined below are controversial, it will be helpful to have at least a plausible candidate for understanding informal validity. So without defending it, let me sketch one such account, calling it the instance account of informal validity. The idea behind it is that a formula is informally valid if and only if all of its natural language instances are true. So a formula like $p \rightarrow \square p$ can be seen not to be informally valid on this account as the sentence "If Obama is president of the US then it is necessary that Obama is president of the US" is a false instance. Of course, it is not completely clear which sentences count as instances, and more generally, what is meant by natural language. But for purposes of illustration, the account is clear enough.

The instance account of informal validity just sketched is reminiscent of the notion of logical truth for logics of quantification proposed in Tarski (1936), which is adapted to modal logics in Williamson (2013, section 3.3). Williamson singles out those formulas $\varphi$ of a propositional modal language such that $\forall p_{1} \ldots \forall p_{n} \varphi$ is true on the intended interpretation, where $p_{1}, \ldots, p_{n}$ are the propositional letters in $\varphi$, and calls them metaphysically universal. While metaphysical universality as defined by Williamson and the instance account of informal validity are closely related, we shouldn't assume that they are the same. This is particularly important in the context of two-dimensional semantics, which provides a number of entities that play some of the roles traditionally associated with propositions. Thus claims about all instances of a formula and its universal closure might come apart in unexpected ways; see the discussion in Fritz (2013, section 4).

Informal validity according to the instance account and the resulting notions of informal soundness and completeness are structurally similar to the standard provability interpretation of modal logic (see Boolos (1993, pp. xxvi-xxvii)). On the provability interpretation, a formula $\varphi$ in the propositional modal language containing only the modal operator $\square$ is called always provable if for every realization $*, \varphi^{*}$ is provable in Peano arithmetic. Here, a realization is a function from proposition letters to first-order formulas in the language of Peano arithmetic. This is extended to complex formulas in the natural way for Boolean connectives and by mapping $\square \psi$ to $\operatorname{Bew}\left(\left\ulcorner\psi^{*}\right\urcorner\right)$, where $\operatorname{Bew}(x)$ is a formula which expresses provability in Peano arithmetic and $\ulcorner\chi\urcorner$ is the numeral of the Gödel number of $\chi$. With this, a modal logic is called arithmetically sound if it contains only formulas that are always provable, and arithmetically complete if it contains all formulas that are always provable.

The analogy to the notions introduced above is straightforward. Instances of modal formulas correspond to the result of applying a realization to a modal formula; having only true instances corresponds to being always provable (i.e., all realizations mapping the formula to one that is provable in Peano arithmetic); and arithmetical soundness and completeness correspond to informal soundness and completeness. Of course, there are also important differences. Since always provable is introduced by a mathematical definition, the question which logic is arithmetically sound and complete is a purely mathematical one. And in fact, one can prove that the modal logic GL is arithmetically sound and complete. Since informally valid is introduced in an informal way, the question which logic is informally sound and complete (i.e., correct) can only be answered by a combination of formal and informal arguments. The aim of this paper is to lay out the basic structure of such a combination. I will first give a sketch of the overall strategy, and in the next two sections fill in the details, considering informal soundness and completeness in turn.

1.3. The Overall Strategy. The strategy followed below is an extension of the argument for the correctness of $\mathbf{S 5}_{\square}$ (the familiar logic $\mathbf{S 5}$ for the modality $\square$ ) presented in 
Williamson (2013, section 3.3). $\mathbf{S 5}_{\square}$ is the normal modal logic axiomatized by $T_{\square}=$ $\square p \rightarrow p$ and $5_{\square}=\diamond p \rightarrow \square \diamond p$, and is sound and strongly complete with respect to the class of frames whose relation is an equivalence relation. Using the terminology of the present paper, Williamson first outlines an argument for the informal soundness of $\mathbf{S 5}_{\square}$ by an induction on the length of proofs in a suitable proof system. He assumes that all of the axioms of the relevant proof system are informally valid and all of its rules preserve informal validity, in the sense that any formula they allow us to derive from informally valid formulas is informally valid as well. It follows by induction that all theorems of $\mathbf{S 5}_{\square}$ are informally valid, and therefore that $\mathbf{S 5}_{\square}$ is informally sound. For informal completeness, Williamson assumes informal soundness, which means that the correct logic contains $\mathbf{S 5} \square$. If we also assume that modus ponens and uniform substitution preserve informal validity, it follows that the correct logic is a quasi-normal extension of $\mathbf{S 5} \square$. He then presents a result characterizing all such extensions, and argues that all proper extensions contain a formula which is not informally valid. Thus $\mathbf{S 5}_{\square}$ is the only remaining candidate for the correct logic, and so a fortiori informally complete.

Below, I will extend this strategy to the logic characterized by MD. In the next section, a proof system will be developed that allows a similar inductive argument for the informal soundness of this logic, and in the following section, a result is proven that characterizes all quasi-normal extensions of this logic in a way that allows for a similar argument for its informal completeness. In both cases, only outlines of the relevant arguments are given. Like the analogous arguments for the correctness of $\mathbf{S 5}_{\square}$, they rely on claims about the informal validity of certain formulas, and I won't argue for all of them. This would require arguing for a specific account of informal validity, as well as taking a stand on a number of philosophically controversial issues; tasks I will not attempt here. Although somewhat incomplete, these considerations still tell us something interesting, as they give us a relatively small list of claims from which we can conclude that the logic characterized by MD is correct. I will also consider which of the relevant claims about informal validity are likely to be controversial, using the instance account of informal validity for concreteness.

The outlined strategy might remind one of Kreisel's squeezing argument in Kreisel (1967). Kreisel argues that classical first-order logic contains all and only intuitively valid formulas. To do so, he first argues that all theorems of classical first-order logic are intuitively valid, by an appeal to the intuitive validity of (and preservation of intuitive validity by) all components of a suitable proof system for classical first-order logic. He then argues that all intuitively valid formulas are theorems of classical first-order logic, by arguing that any formula that is false in some set-theoretic structure is not intuitively valid. The crucial technical premise of the argument is the fact that classical first-order logic is both characterized by the relevant proof system and the semantics given by set-theoretic structures (i.e., Gödel's completeness theorem). If we assume that what Kreisel calls intuitive validity is the same as what I have been calling informal validity, then Kreisel's argument can naturally be described in the terminology introduced above. The first step of the argument is an argument for informal soundness of classical first-order logic, and the second step is an argument for its informal completeness. And more abstractly, we are doing the same as what Kreisel attempts, namely to take an informal notion (suitably clarified, as argued in Smith (2011)) and use formal results to prove that certain informal constraints motivated by this notion pin down a formal construct uniquely.

Could we also call the strategy that will be followed below a squeezing argument? This would not be natural. The term squeezing is appropriate in Kreisel's case, since his argument is of the following form: We have two mathematically defined notions $D$ and $V$ 
(deducible and formally valid formulas), and an informally introduced notion $I$ (intuitively valid formulas). We argue informally that $I$ contains $D$ and $V$ contains $I$. We squeeze by proving formally that $D$ and $V$ coincide in extension, thus showing that all three coincide in extension. While the informal soundness part of the present strategy is quite similar to the analogous step in the squeezing argument, the informal completeness part is rather different, as it is based on a syntactic characterization of extensions of the relevant logic, rather than a formal semantics for the logic. And this makes a big difference when we consider extending the argument to consequence relations. Kreisel's semantic method straightforwardly extends to informal/intuitive validity of arguments rather than formulas, and thus to logics understood as consequence relations. In contrast, the syntactic method used here does not immediately extend to such a setting. This can be seen from the fact that there are multiple consequence relations extending the consequence relation of $\mathbf{S 5} \square$ which agree on what follows from no premises, therefore additional assumptions are needed to extend the present strategy to consequence relations; see Williamson (2013, pp. 111-114). Such extensions won't be considered in the following.

\$2. Informal Soundness. To show that a logic is informally sound, we have to argue that all of its theorems are informally valid. As noted above, a natural way of doing so in the case of $\mathbf{S 5}_{\square}$ uses a standard proof system resulting from its definition as the normal modal logic axiomatized by $T_{\square}$ and $5_{\square}$. One such system contains as axioms the propositional tautologies, $K_{\square}=\square(p \rightarrow q) \rightarrow(\square p \rightarrow \square q)$, $T_{\square}$ and $5_{\square}$; and as rules modus ponens, uniform substitution and the rule of generalization for $\square$ (if $\varphi$ is a theorem so is $\square \varphi$ ). If we can show that all of these axioms are informally valid and the three rules preserve informal validity, then it follows by induction that all theorems of $\mathbf{S 5}_{\square}$ are informally valid. Of course, showing that the premises are true may be difficult in some cases. But at least, we now only have to consider a limited number of axioms and rules (finite if we replace the propositional tautologies by one of their finite axiomatizations), rather than an infinite set of theorems.

To give a similar argument for the informal soundness of the logic characterized by MD, we have to develop a syntactic characterization for it. This is done in a natural way in the next section. Unfortunately, we will see that the characterization obtained this way is not suitable for the kind of inductive argument for informal soundness we are interested in here. Therefore, an alternative proof system of the right shape will be derived in the subsequent section.

2.1. Axioms for the logic characterized by MD. As mentioned in Fritz (2013), the logic of MD (i.e., the logic characterized by MD, which is the set of formulas valid in every frame in MD) is not normal. Therefore, standard methods for finding an axiomatization for the logic of a class of frames will not work for the logic characterized by MD. But we can roughly follow the strategy used, e.g., in Vlach (1973) and Crossley \& Humberstone (1977), by first finding an axiomatization of the logic of the class of matrix frames $\mathrm{M}$ and then syntactically deriving a second logic from this, which can then be proven sound and complete with respect to the class of matrix FWDEs MD. In doing so, I will make use of some standard definitions and results in modal logic, which can be found, e.g., in Blackburn et al. (2001), whose terminology and notation I largely adopt. First, we show that the logic characterized by $\mathbf{M}$ is $\mathbf{2} \mathbf{D g}$, which is defined as follows:

DEFINITION 2.1. Let 2Dg be the normal modal logic axiomatized by the following formulas: 


$\begin{array}{llll}T_{\square} & \square p \rightarrow p & I 2 & @ p \rightarrow \square @ p \\ 5 \square & \diamond p \rightarrow \square \diamond p & 4_{A} & A p \rightarrow A A p \\ D_{@} & @ p \rightarrow \neg @ \neg p & 5_{A} & C p \rightarrow A C p \\ D_{c} @ & \neg @ \neg p \rightarrow @ p & I 3 & A p \rightarrow @ p \\ I 1 & \square p \rightarrow @ p & I 4 & A(@ p \rightarrow p)\end{array}$

These are just the axioms in Davies \& Humberstone (1980) for the logic of necessity and actuality, plus four axioms concerning apriority and its relation to actuality. All of these axioms are Sahlqvist formulas, so the conditions on frames they express can be calculated by the Sahlqvist-van Benthem algorithm. The following table lists the axioms of $\mathbf{2 D g}$ and their local frame correspondents, i.e., formulas of predicate logic expressing conditions that are satisfied by a point $w$ of a frame if and only if the corresponding axiom is valid in that frame at that point:

\begin{tabular}{|c|c|c|}
\hline$T_{\square}$ & $\square p \rightarrow p$ & $w R_{\square} w$ \\
\hline $5 \square$ & $\diamond p \rightarrow \square \diamond p$ & $\forall v u\left(\left(w R_{\square} v \wedge w R_{\square} u\right) \rightarrow v R_{\square} u\right)$ \\
\hline$D_{@}$ & $@ p \rightarrow \neg @ \neg p$ & $\exists v(w R @ v)$ \\
\hline$D_{c} @$ & $\neg @ \neg p \rightarrow @ p$ & $\forall v u\left(\left(w R_{@} v \wedge w R_{@} u\right) \rightarrow v=u\right)$ \\
\hline$I 1$ & $\square p \rightarrow @ p$ & $\forall v\left(w R_{@} v \rightarrow w R_{\square} v\right)$ \\
\hline$I 2$ & $@ p \rightarrow \square @ p$ & $\forall v u\left(\left(w R_{\square} v \wedge v R_{@} u\right) \rightarrow w R_{@} u\right)$ \\
\hline $4_{A}$ & $A p \rightarrow A A p$ & $\forall v u\left(\left(w R_{A} v \wedge v R_{A} u\right) \rightarrow w R_{A} u\right)$ \\
\hline $5_{A}$ & $C p \rightarrow A C p$ & $\forall v u\left(\left(w R_{A} v \wedge w R_{A} u\right) \rightarrow v R_{A} u\right)$ \\
\hline$I 3$ & $A p \rightarrow @ p$ & $\forall v\left(w R_{@} v \rightarrow w R_{A} v\right)$ \\
\hline$I 4$ & $A(@ p \rightarrow p)$ & $\forall v\left(w R_{A} v \rightarrow v R_{@} v\right)$ \\
\hline
\end{tabular}

Furthermore, the fact that the axioms are Sahlqvist formulas implies that 2Dg is strongly complete with respect to $\mathrm{Fr}_{\mathbf{2 D g}}$, the class of 2Dg-frames (frames in which $\mathbf{2 D g}$ is valid):

THEOREM 2.2. 2Dg is sound and strongly complete with respect to $\mathrm{Fr}_{\mathbf{2 D g}}$.

Proof. By the Sahlqvist completeness theorem; see, e.g., Blackburn et al. (2001, Theorem 4.42).

To prove that $2 \mathrm{Dg}$ is sound and strongly complete with respect to M, I will first show that a set of formulas is satisfiable on $\mathrm{Fr}_{\text {2Dg }}$ if and only if it is satisfiable on a class of frames $\mathrm{R}$ which is contained in $\mathrm{Fr}_{2 \mathrm{Dg}}$ and contains M. I will then show that a set of formulas is satisfiable on $R$ if and only if it is satisfiable on $M$. These claims will be established by proving that $R$ is the class of point-generated subframes of $\mathbf{2 D g}$-frames, as well as the class of bounded morphic images of matrix frames. The desired claims about satisfiability follow from these structural connections by well-known invariance results. Since a logic is sound and strongly complete with respect to a class of frames if and only if the sets of formulas consistent in the logic are exactly the sets satisfiable on the class of frames, the completeness of $\mathbf{2 D g}$ with respect to $\mathrm{M}$ follows by Theorem 2.2. In a slightly different form, the intermediate class of frames $R$ is used in Restall (2012), so I will call them Restall frames. Calling a relation a function if it is serial and functional, and writing $\operatorname{im}(R)$ for the image of a relation $R$, they can be defined as follows:

DEFINITION 2.3. A Restall frame is a frame $\mathfrak{F}=\left\langle W, R_{\square}, R_{@}, R_{A}\right\rangle$ such that

- $R_{\square}$ is an equivalence relation,

- $R_{@}$ is a function such that 
(i) if $w R_{@} v$ then $w R_{\square} v$ and

(ii) $R_{@}$ maps any two $R_{\square}$-related points to the same point, and

- $w R_{A} v$ iff $v \in \operatorname{im}\left(R_{@}\right)$.

Let $\mathrm{R}$ be the class of Restall frames.

LEMMA 2.4. Every Restall frame is a point-generated subframe of a 2Dg-frame.

Proof. Consider any Restall frame $\mathfrak{F}=\left\langle W, R_{\square}, R_{@}, R_{A}\right\rangle$. We first show that for any $w \in W, \mathfrak{F}_{w}$ (the subframe of $\mathfrak{F}$ generated by $w$ ) is $\mathfrak{F}$ itself. Consider any $v \in W$. Since $R_{@}$ is serial, there is a $u \in W$ such that $v R_{@} u$. So also $v R_{\square} u$, and by symmetry of $R_{\square}, u R_{\square} v$. It is also the case that $u \in \operatorname{im}\left(R_{@}\right)$, so $w R_{A} u$. It follows from $w R_{A} u$ and $u R_{\square} v$ that $v$ is in $\mathfrak{F}_{w}$. As $v$ was chosen arbitrarily, $\mathfrak{F}_{w}=\mathfrak{F}$.

To show that $\mathfrak{F}$ is itself a $\mathbf{2 D g}$-frame, it suffices to go through the axioms of $\mathbf{2 D g}$ and verify that the properties defined by them are satisfied by Restall frames. This is straightforward for all axioms except I4. For this, we can reason as follows: let $v \in$ $\operatorname{im}\left(R_{A}\right)$. Then $v \in \operatorname{im}\left(R_{@}\right)$, so there is a $u$ such that $u R_{@} v$. It follows that $u R_{\square} v$, and therefore that $R_{@}$ must map $u$ and $v$ to the same point. So $v R_{0} v$.

The next result will make use of the fact that the formula $N 1=A p \rightarrow \square A p$, which will play an important role in the following, is a theorem of 2Dg. This is shown in the following lemma:

LEMma 2.5. $\vdash_{2 \mathrm{Dg}} A p \rightarrow \square A p$.

Proof. By the following derivation:

$\begin{array}{lll}\text { (1) } C p \rightarrow A C p & 5_{A} \\ (2) A C p \rightarrow @ C p & I 3 \\ (3) C p \rightarrow @ C p & (1),(2) \\ (4)-\neg @ \neg A \neg p \rightarrow A \neg p & (3) \\ (5) @ A p \rightarrow A p & D_{@},(4) \\ (6) \square @ A p \rightarrow \square A p & K_{\square},(5) \\ (7) @ A p \rightarrow \square @ A p & I 2 \\ (8) \text { @ } A p \rightarrow \square A p & (6),(7) \\ (9) A p \rightarrow A A p & 4_{A} \\ (10) A A p \rightarrow @ A p & I 3 \\ (11) A p \rightarrow @ A p & (9),(10) \\ (12) & A p \rightarrow \square A p & (8),(11)\end{array}$

Note that $A p \rightarrow \square A p$ is also a Sahlqvist formula, and that it is therefore straightforward to calculate that it has the following local frame correspondent: $\forall v u\left(\left(w R_{\square} v \wedge v R_{A} u\right) \rightarrow\right.$ $\left.w R_{A} u\right)$.

LEMMA 2.6. Every point-generated subframe of a 2Dg-frame is a Restall frame.

Proof. Consider any 2Dg-frame $\mathfrak{F}=\left\langle W, R_{\square}, R_{@}, R_{A}\right\rangle$ and $w \in W$. Let $\mathfrak{F}_{w}=$ $\left\langle W^{\prime}, R_{\square}^{\prime}, R_{@}^{\prime}, R_{A}^{\prime}\right\rangle$ be the subframe generated by $w$. Since validity is preserved under taking generated subframes, all of the axioms of 2Dg are valid in $\mathfrak{F}_{w}$.

Using $T_{\square}$ and $5_{\square}$, it is routine to show that $R_{\square}^{\prime}$ is an equivalence relation. Likewise, $D_{@}$ and $D_{c} @$ imply that $R_{@}^{\prime}$ is a function, and $I 1$ and $I 2$ that it satisfies conditions (i) and (ii). 
To show that $v R_{A}^{\prime} u$ if and only if $u \in \operatorname{im}\left(R_{@}^{\prime}\right)$, assume first that $v R_{A}^{\prime} u$. Then by $I 4$, $u R_{@}^{\prime} u$, and so $u \in \operatorname{im}\left(R_{@}^{\prime}\right)$. It only remains to show that if $u \in \operatorname{im}\left(R_{@}^{\prime}\right)$, then $v R_{A}^{\prime} u$. We will do so in the rest of this proof, adopting the notation to write $R[Y]$ for the image of a set $Y$ under a relation $R$. Let $w^{\prime}$ be the element of $W^{\prime}$ such that $w R_{@}^{\prime} w^{\prime}$. The existence and uniqueness of this point are guaranteed by the fact that $R_{@}^{\prime}$ is a function. We first prove a preliminary claim:

Claim 1: $W^{\prime}=X$, where $X=R_{\square}\left[R_{A}[\{w\}]\right]$. Clearly $X \subseteq W^{\prime}$. We first show that $w \in X$, and then that $X$ is closed under each of the relations, that is, that $R_{\nabla}[X] \subseteq X$ for every modality $\nabla$.

$w R_{@} w^{\prime}$, so both $w R_{A} w^{\prime}$ and $w R_{\square} w^{\prime}$. Since $R_{\square}$ is symmetric, $w^{\prime} R_{\square} w$, and therefore $w \in X$. Assume that $v \in R_{\square}[X]$. Then there is a $u \in X$ such that $u R_{\square} v$. Since $u \in X$, there is a $u^{\prime} \in W$ such that $w R_{A} u^{\prime}$ and $u^{\prime} R_{\square} u$. By transitivity of $R_{\square}, u^{\prime} R_{\square} v$, and so $v \in X$. Assume that $v \in R_{@}[X]$. Then there is a $u \in X$ such that $u R_{@} v$. By I1, $R_{@} \subseteq R_{\square}$, so $u R_{\square} v$. That $v \in X$ follows by transitivity of $R_{\square}$ as before. Assume that $v \in R_{A}[X]$. Then there is a $u \in X$ such that $u R_{A} v$, and therefore a $u^{\prime} \in W$ such that $w R_{A} u^{\prime}$ and $u^{\prime} R_{\square} u$. By Lemma 2.5, it follows that $u^{\prime} R_{A} v$, and so by transitivity of $R_{A}$ that $w R_{A} v$. Since $R_{\square}$ is reflexive, $v \in X$. This concludes the proof of claim 1 .

Now consider any $u \in \operatorname{im}\left(R_{@}^{\prime}\right)$ and $v \in W^{\prime}$. We have to prove that $v R_{A}^{\prime} u$. We do this by first proving that $v R_{A}^{\prime} w^{\prime}$ and then that $w^{\prime} R_{A}^{\prime} u$.

Claim 2: $v R_{A}^{\prime} w^{\prime}$. Since $v \in W^{\prime}$, it follows from claim 1 that there is a $v^{\prime} \in W^{\prime}$ such that $w R_{A}^{\prime} v^{\prime}$ and $v^{\prime} R_{\square}^{\prime} v$. By symmetry of $R_{\square}^{\prime}, v R_{\square}^{\prime} v^{\prime}$. Since $w R_{@}^{\prime} w^{\prime}$, by $I 3$ also $w R_{A}^{\prime} w^{\prime}$. So since $R_{A}^{\prime}$ is Euclidean, $v^{\prime} R_{A}^{\prime} w^{\prime}$. By Lemma 2.5, it follows that $v R_{A}^{\prime} w^{\prime}$.

Claim 3: $w^{\prime} R_{A}^{\prime} u$. Since $u \in W^{\prime}$, there is a $u^{\prime} \in W^{\prime}$ such that $w R_{A}^{\prime} u^{\prime}$ and $u^{\prime} R_{\square}^{\prime} u$. As we've seen before, $w R_{A}^{\prime} w^{\prime}$, so since $R_{A}^{\prime}$ is Euclidean, $w^{\prime} R_{A}^{\prime} u^{\prime}$. Also $u \in \operatorname{im}\left(R_{@}^{\prime}\right)$, so there is a $u^{\prime \prime} \in W^{\prime}$ such that $u^{\prime \prime} R_{@}^{\prime} u$. By I1 also $u^{\prime \prime} R_{\square}^{\prime} u$, and with the fact that $R_{\square}^{\prime}$ is an equivalence relation, $u^{\prime} R_{\square}^{\prime} u^{\prime \prime}$. So by $\mathrm{I} 2$, it follows that $u^{\prime} R_{@}^{\prime} u$, and with $\mathrm{I} 3, u^{\prime} R_{A}^{\prime} u$. Since $R_{A}^{\prime}$ is transitive, $w^{\prime} R_{A}^{\prime} u$.

By transitivity of $R_{A}^{\prime}$, it follows from claims 2 and 3 that $v R_{A}^{\prime} u$.

With these Lemmas, it follows that 2Dg is sound and complete with respect to $\mathrm{R}$. Without much effort, we could use Restall (2012, Theorem 8) to conclude that 2Dg is sound and complete with respect to $\mathrm{M}$. But in order to bring out the structural connections between the classes of frames, we continue with our initial proof strategy:

\section{LEMMA 2.7. Every bounded morphic image of a matrix frame is a Restall frame.}

Proof. By checking the conditions on Restall frames, one can verify that matrix frames are Restall frames. With this, the claim follows from the fact that $\mathrm{R}$ is closed under taking bounded morphic images, which is routine to prove.

LEMMA 2.8. Every Restall frame is a bounded morphic image of a matrix frame.

Proof. Let $\mathfrak{F}=\left\langle W, R_{\square}, R_{@}, R_{A}\right\rangle$ be a Restall frame. We proceed by constructing a matrix frame $\mathfrak{F}^{\prime}$ and a surjective bounded morphism $f$ from $\mathfrak{F}^{\prime}$ to $\mathfrak{F}$. I will use the following notation: $[x]_{E}$ is the equivalence class of $x$ under the equivalence relation $E$. For a relation $R$ that is a function, $R(x)$ is the unique $y$ such that $x R y$.

Let $\mathfrak{F}^{\prime}$ be the matrix frame whose set of points is $W \times W$, and for every $w \in W$, let $\alpha_{w}: W \rightarrow[w]_{R_{\square}}$ be a surjection such that $\alpha_{w}(w)=R_{@}(w)$. Such surjections exist for cardinality reasons and the fact that $R_{@}$ is a function for which $R_{@} \subseteq R_{\square}$ holds. We define $f: W \times W \rightarrow W$ by $f(\langle w, v\rangle)=\alpha_{v}(w)$. 
We can prove that $f$ is a surjective bounded morphism from $\mathfrak{F}^{\prime}$ to $\mathfrak{F}$. To show that it is a bounded morphism, one only has to go through the modalities and check the forth and back conditions. For surjectivity, consider any $w \in W$. Then $\alpha_{w}: W \rightarrow[w]_{R_{\square}}$ is a surjective function. Therefore, there is a $v \in W$ such that $\alpha_{w}(v)=w$. So $f(\langle v, w\rangle)=w$.

THEOREM 2.9. 2Dg is sound and strongly complete with respect to $\mathrm{M}$.

Proof. The preceding lemmas establish that Restall frames are both the point-generated subframes of 2Dg-frames as well as the bounded morphic images of matrix frames. Since truth is invariant under taking generated submodels (see Blackburn et al. (2001, Proposition 2.6)) as well as bounded morphisms between models (see Blackburn et al. (2001, Proposition 2.14)), one can show with standard arguments that a set of formulas is satisfiable on $\mathrm{Fr}_{\text {2Dg }}$ if and only if it is satisfiable on $R$, and that this is the case if and only if it is satisfiable on M. With Theorem 2.2, the claim follows.

In the same way in which Crossley \& Humberstone (1977) derive their logic of realworld validity from their logic of general validity, we can use 2Dg to define a logic 2D, and infer from Theorem 2.9 that it is sound and complete with respect to MD. Since MD is the formal semantics that captures two-dimensional semantics, this completeness result means that the following definition of $\mathbf{2 D}$ gives us a syntactic characterization of our logic of two-dimensional semantics:

DEFINITION 2.10. $\vdash_{2 D} \varphi$ if and only if $\vdash_{\mathbf{2 D g}} @ \varphi$.

THEOREM 2.11. 2D is sound and strongly complete with respect to MD.

Proof. We show that any set of formulas is 2D-consistent if and only if it is satisfiable on MD. Note that it is straightforward to verify that 2D is quasi-normal. First, let $\Gamma$ be a 2D-inconsistent set. Then there are $\varphi_{1}, \ldots, \varphi_{n} \in \Gamma$ such that $\vdash_{2 D} \neg \bigwedge_{i \leq n} \varphi_{i}$. So by definition of 2D, $\vdash_{2 D g} @ \neg \bigwedge_{i \leq n} \varphi_{i}$. Consider any matrix FWDE $\mathcal{F}$ with set of points $W$, relation $R_{@}$, and distinguished points $D$, and let $w \in D$. By the soundness of $2 \mathbf{D g}$, $\mathcal{F}, w \Vdash @ \neg \bigwedge_{i \leq n} \varphi_{i}$. Since $w R_{@} w$, also $\mathcal{F}, w \Vdash \neg \bigwedge_{i \leq n} \varphi_{i}$. Hence $\Gamma$ is not satisfiable on MD.

Now, let $\Gamma$ be a set that is not satisfiable on MD. Assume for contradiction that $\Gamma^{@}=$ $\{@ \varphi: \varphi \in \Gamma\}$ is satisfiable on $\mathrm{M}$. Then there is a matrix frame $\mathfrak{F}$ with set of points $W$ and relation $R_{@}$, and a point $w \in W$ such that $\Gamma^{@}$ is satisfiable in $\mathfrak{F}$ at $w$. Since $R_{@}$ is a function, there is a $v \in W$ such that $w R_{@} v$, so $\Gamma$ is satisfiable in $\mathfrak{F}$ at $v$. But then $v \in \operatorname{im}\left(R_{@}\right)$, so $\Gamma$ is satisfiable on MD. , so $\Gamma^{@}$ is not satisfiable on M. By strong completeness of 2Dg it follows that $\Gamma^{@}$ is $\mathbf{2 D g}$-inconsistent, and so that there are $\varphi_{1}, \ldots, \varphi_{n} \in \Gamma$ such that $\vdash_{2 D g} \neg \bigwedge_{i \leq n} @ \varphi_{i}$. Since $D_{@}$ and $D_{c} @$ are theorems of 2Dg, @ distributes over Boolean connectives in 2Dg. Therefore $\vdash_{2 D g} @ \neg \bigwedge_{i \leq n} \varphi_{i}$, and so by definition of $\mathbf{2 D}, \vdash_{2 \mathbf{D}} \neg \bigwedge_{i \leq n} \varphi_{i}$. Hence $\Gamma$ is $\mathbf{2 D}$-inconsistent.

2.2. An Alternative Proof System for 2D. With this completeness result, the definition of 2D gives us a syntactic characterization of the logic characterized by MD (which I will just call 2D from now on). But as announced, the inductive way of arguing for informal soundness demonstrated on $\mathbf{S 5}_{\square}$ is not applicable to it, simply because $\mathbf{2 D}$ is not defined as the set of formulas derivable from a set of axioms using a set of rules. One might think that the specific construction of $\mathbf{2 D}$ at least allows us to argue similarly. In particular, one might propose to argue first that all theorems of 2Dg are informally valid, analogously to the above argument for the informal soundness of $\mathbf{S 5}_{\square}$, and then to infer from this that the theorems of $\mathbf{2 D}$ are informally valid. Indeed, the second step seems easy: using 
Definition 2.10, we can show that $2 \mathrm{D}$ is the logic of the proof system that contains $T_{@}=$ @ $p \rightarrow p$ and all theorems of $\mathbf{2 D g}$ as axioms, and modus ponens and uniform substitution as rules. (The logic of a proof system is the set of formulas derivable in it.) Thus given the assumption that $\mathbf{2 D g}$ is informally valid, the informal validity of 2D follows by induction from the premise that $T_{@}$ is informally valid and modus ponens and uniform substitution preserve informal validity.

However, the first step of the proposal is problematic. 2Dg is defined as the normal modal logic axiomatized by some formulas, which means that the rules of its standard proof system include the rule of generalization for $\square$. Therefore, we would have to argue that if $\varphi$ is an informally valid formula, so is $\square \varphi$. But this is likely not to be the case, since at least on the instance account of informal validity, @ $p \rightarrow p$ is informally valid but $\square(@ p \rightarrow p)$ is not, which is reflected in 2D in the sense that the former is a theorem of 2D, but the latter is not. (Note that there is no inconsistency in claiming that generalization for $\square$ preserves informal validity in the context of $\mathbf{S 5}_{\square}$ while it fails to do so in the context of 2D.) The reason why the inductive argument for informal validity does not work here is that in contrast to $\mathbf{2 D}$, being a theorem of $\mathbf{2 D g}$ is not supposed to track being informally valid. As 2Dg is a proper subset of 2D, it can be seen as tracking a stronger property, which entails having an informally valid necessitation.

So to give an inductive argument for the informal soundness $2 \mathbf{D}$, we need to develop an alternative proof system for it that has the required form. Doing so is the task of this section. The alternative proof system will be constructed in a way that allows us to outline an argument for the informal soundness of $\mathbf{2 D}$ in several steps, roughly speaking by first considering the logics of the individual modalities, and then the interactions between the modalities. As noted in Fritz (2013), 2D contains as the logic of necessity and actuality the logic in Crossley \& Humberstone (1977) according to real-world validity (which I will call Act), in the sense that it is a conservative extension of that logic. Also, 2D contains as the logic of apriority $\mathbf{S 5}_{A}$ ( $\mathbf{S 5}$ for the modality $A$ ), in the sense that it is also a conservative extension of that logic. So given that we can argue that $\mathbf{A c t}$ and $\mathbf{S 5} \mathbf{5}_{A}$ are informally sound, we only have to argue for the informal validity of the principles of 2D that are not already contained in these two logics. If we can specify these in a finite way, then we can argue inductively for the informal soundness of $\mathbf{2 D}$, at least if we have previously argued that Act and $\mathbf{S 5}_{A}$ are informally sound.

I start by formally defining Act. First, Actg is defined to be the normal modal logic axiomatized by the formulas $T_{\square}, 5_{\square}, D_{@}, D_{c} @, I 1$, and $I 2$, which were given above. (As noted there, this is the axiomatization from Davies \& Humberstone (1980); the one in Crossley \& Humberstone (1977) contains an additional axiom, which can be shown to be redundant.) From this, we derive Act by postulating that $\vdash_{\text {Act }} \varphi$ if and only if $\vdash_{\text {Actg }} @ \varphi$.

2D is quasi-normal, so it is clear that it has to contain the theorems of $\mathbf{K}$ and be closed under modus pones and uniform substitution. Thus in constructing our proof system, we can just add these as axioms and rules to the theorems of Act and $\mathbf{S 5}_{A}$. It only remains to add some axioms and rules that encode the interactions between the modalities in a finite way. Here is one way of doing so:

Definition 2.12. Let $P_{2 \mathbf{D}}$ be the proof system containing as rules modus ponens, uniform substitution, and generalization for $A$, and as axioms the theorems of $\mathbf{K}$, Act and $\mathbf{S 5}_{A}$, as well as the following formulas:

$\begin{array}{llll}\square 4_{A} & \square(A p \rightarrow A A p) & \square D_{A} & \square(A p \rightarrow C p) \\ \square 5_{A} & \square(C p \rightarrow A C p) & N 1 & A p \rightarrow \square A p\end{array}$


To show that the logic of $P_{\mathbf{2 D}}$ is in fact $\mathbf{2 D}$, we prove that $\square I 3=\square(A p \rightarrow @ p)$ is derivable in $P_{\mathbf{2 D}}$. To do this, we first show that $C p \rightarrow \square C p$ is derivable as well:

LEMMA 2.13. $\vdash_{P_{2 \mathbf{D}}} C p \rightarrow \square C p$.

Proof. By the following derivation:
(1) $\square(A C p \rightarrow C C p) \quad \square D_{A}$
(2) $\square(A \neg p \rightarrow A A \neg p) \quad \square 4_{A}$
(3) $\square(C C p \rightarrow C p) \quad$ (2), $\mathbf{K}$
(4) $\square(A C p \rightarrow C p) \quad$ (1), (3), $\mathbf{K}$
(5) $\square A C p \rightarrow \square C p \quad$ (4), $\mathbf{K}$
(6) $A C p \rightarrow \square A C p \quad N 1$
(7) $C p \rightarrow A C p \quad \square 5_{A}, T_{\square}$
(8) $C p \rightarrow \square C p \quad$ (7), (6), (5)

LEMMA 2.14. $\vdash_{P_{2 D}} \square I 3$.

Proof. By the following derivation:

$\begin{array}{lll}\text { (1) } & p \rightarrow C p & T_{A} \\ \text { (2) } p \rightarrow \square C p & (1), \text { Lemma } 2.13 \\ \text { (3) } \diamond A p \rightarrow p & (2) \\ \text { (4) } p \rightarrow \square @ p & \text { Act } \\ \text { (5) } \diamond A p \rightarrow \square @ p & \text { (3), (4) } \\ \text { (6) } \square(A p \rightarrow @ p) & \text { (5), K }\end{array}$

With this lemma, we can prove the desired result:

THEOREM 2.15. The logic of $P_{\mathbf{2 D}}$ is $\mathbf{2 D}$.

Proof. We first show by induction on the length of proofs in $P_{\mathbf{2 D}}$ that its logic is included in 2D. Since 2D is a quasi-normal extension of both Act and $\mathbf{S 5} \mathbf{5}_{A}$, it contains the theorems of $\mathbf{K}$, Act and $\mathbf{S 5}_{A}$ and is closed under modus ponens and uniform substitution. It is straightforward to show that $\mathbf{2 D}$ contains the other axioms and is closed under generalization for $A$ by semantic arguments using MD.

To show that $2 \mathrm{D}$ is included in $P_{\mathbf{2 D}}$, we first prove by induction on the construction of $\mathbf{2 D g}$ that $\varphi \in \mathbf{2 D g}$ implies $\vdash_{P_{2 \mathbf{D}}} \square \varphi$. If $\varphi$ is one of the axioms of Actg, this follows from the fact that Actg $\subseteq$ Act. If it is $4_{A}$ or $5_{A}$, its necessitation is an axiom of $P_{\mathbf{2 D}}$. For I3, we have shown in Lemma 2.14 that $\vdash_{P_{2 \mathrm{D}}} \square I 3$. The case of I4 follows by @ $p \rightarrow$ $p \in$ Act, the rule of generalization for $A$ and $N 1$. The rules of modus ponens and uniform substitution are straightforward by induction. Generalization for $\square$ can be dealt with using 4ロ, and generalization for @ using $I 1$ and I2, which are all in Act. Finally, the case of generalization for $A$ follows by $T_{\square}$, generalization for $A$ and $N 1$.

Now consider any $\varphi \in$ 2D. By definition, @ $\varphi \in \mathbf{2 D g}$, so by the claim just proven, $\vdash_{P_{2 \mathbf{D}}} \square @ \varphi$. Since $\square @ p \rightarrow p \in$ Act, it follows that $\vdash_{P_{2 \mathbf{D}}} \varphi$.

2.3. Outline of an Informal Soundness Argument. By Theorem 2.15, we can use $P_{2 D}$ to argue for the informal soundness of 2D in the same way we argued for the informal soundness of $\mathbf{S 5}_{\square}$ : First, we show that all axioms of $P_{\mathbf{2 D}}$ are informally valid, and then that 
its rules preserve informal validity. The first set of axioms of $P_{\mathbf{2 D}}$ are the theorems of $\mathbf{K}$, Act and $\mathbf{S 5}_{A}$, so we first have to show that these logics are informally sound.

Setting up such an argument is easiest for $\mathbf{S 5}_{A}$. Analogous to the case for $\mathbf{S 5}_{\square}$, since $\mathbf{S 5}_{A}$ is the normal modal logic axiomatized by $T_{A}$ and $5_{A}$, we can argue for its informal soundness by showing that the relevant axioms are informally valid and the relevant rules preserve informal validity. As noted in Williamson (2013, p. 110) for the analogous case of $\mathbf{S 5}_{\square}$, we can also replace the rule of generalization for $A$ by the axiom $4_{A}$, if we replace the propositional tautologies by their necessitations.

We cannot argue this straightforwardly for Act, since it is derived from Actg in the way 2D is derived from 2Dg. But we can do so using the following alternative proof system $P_{\text {Act }}$ for Act:

Definition 2.16. P $P_{\text {Act }}$ is the proof system containing the rules of modus ponens and uniform substitution and as axioms the necessitations of propositional tautologies and the following:

$$
\begin{array}{llll}
K_{\square} & \square(p \rightarrow q) \rightarrow(\square p \rightarrow \square q) & \square D_{@} & \square(@ p \rightarrow \neg @ \neg p) \\
T_{\square} & \square p \rightarrow p & \square D_{c} @ \square(\neg @ \neg \rightarrow @ p) \\
4 \square & \square p \rightarrow \square \square p & \square I 1 & \square(\square p \rightarrow @ p) \\
5 \square & \diamond p \rightarrow \square \diamond p & \square I 2 & \square(@ p \rightarrow \square @ p) \\
\square K_{@} & \square(@(p \rightarrow q) \rightarrow(@ p \rightarrow @ q)) & T_{@} & @ p \rightarrow p
\end{array}
$$

Proposition 2.17. The logic of $P_{\text {Act }}$ is Act.

Proof. That Act includes the logic of $P_{\text {Act }}$ is straightforwardly proven by induction. The converse direction can be shown analogously to the corresponding direction in the proof of Theorem 2.15, using the proofs in Williamson $(2013$, p. 110, fn. 36) for the cases of $K_{\square}, T_{\square}$ and $5_{\square}$.

Thus, we can argue for the informal soundness of Act by arguing that the axioms of $P_{\text {Act }}$ are informally valid and the rules $P_{\text {Act }}$ preserve informal validity. Similar to the two variants of providing a proof system for $\mathbf{S 5}_{A}$ discussed above, we can also replace the axiom $4 \square$ in $P_{\text {Act }}$ by a rule, which now has to be a restricted rule of generalization for $\square$, namely the rule which says that if $\varphi$ is a theorem not containing any other modality than $\square$, then $\square \varphi$ is a theorem as well (and we can use the propositional tautologies rather than their necessitations). The resulting proof system is very similar to the proof system for a temporal logic with the indexical operator "now" presented in Prior (1968, p. 113).

Arguing for the informal soundness of $\mathbf{K}$ might also seem difficult, since its standard axiomatization makes use of the problematic rule of generalization for $\square$. But we can dispense with this rule by using two axiom schemas. Let $*$ Prop be the axiom schema whose instances are the formulas $\nabla \varphi$ where $\nabla$ is a finite sequence of modal operators (i.e., $\square$, @ or $A$ ) and $\varphi$ is a propositional tautology. Let $* K_{\text {? }}$ be the axiom schema whose instances are the formulas $\nabla K_{\nabla}$ where $\nabla$ is a finite sequence of modal operators and $\nabla$ is a modal operator. We can show that $\mathbf{K}$ is the logic of the proof system containing the axiom schemas $*$ Prop and $* K_{\text {? }}$ and the rules of modus ponens and uniform substitution. (See Williamson (2013, p. 98) who presents a variant of this that also dispenses with the rule of uniform substitution.) Thus the informal soundness of $\mathbf{K}$ follows from the informal validity of the instances of the two schemas and the preservation of informal validity by the two rules. Although this is an infinite axiomatization, the axioms are instances of two schemas, which means that a general argument for the informal validity all of them is feasible. 
It remains to argue for the informal validity of the additional axioms of $P_{\mathbf{2 D}}$ and to argue that the additional rule of $P_{2}$ D preserves informal validity. That is, we have to argue that $\square 4_{A}, \square 5_{A}, \square D_{A}$ and $N 1$ are informally valid and generalization for $A$ preserves informal validity - the rules of modus ponens and uniform substitution have already been considered for the present language in the discussion of $\mathbf{K}$. With Theorem 2.15, an inductive argument shows as in the case for $\mathbf{S 5}_{\square}$ that this implies that all theorems of $\mathbf{2 D}$ are informally valid, and that 2D is therefore informally sound.

This concludes the outline of the argument for the informal soundness of 2D. We now have a finite list of axioms or axiom schemas and rules, which can be used to argue that 2D is informally sound. In fact, we can use this list to give yet another proof system for $\mathbf{2 D}$ (eliminating some obvious redundancies):

DEFINITION 2.18. Let $P_{2 \mathbf{D}}^{\prime}$ be the proof system containing as rules modus ponens, uniform substitution and generalization for $A$; and as axioms the instances of $*$ Prop and $* K_{\text {? }}$, as well as $T_{\square}, 4_{\square}, 5_{\square}, \square D_{@}, \square D_{c} @, \square I 1, \square I 2, T_{@}, T_{A}, \square 4_{A}, \square 5_{A}, \square D_{A}$, and $N 1$.

Proposition 2.19. The logic of $P_{\mathbf{2 D}}^{\prime}$ is $\mathbf{2 D}$.

Proof. By inductions on the length of proofs in $P_{2 \mathbf{D}}$ and $P_{2 \mathbf{D}}^{\prime}$.

So far, I have only outlined how one could give an inductive argument for the informal soundness of 2D, without considering the particular claims needed for this in any detail. I will now consider which ones of them are likely to be controversial. For concreteness, I will use the instance account of informal validity.

2.4. Arguing for the Premises. Let me start with the claims needed to argue that $\mathbf{K}$ is informally sound. Assuming classical propositional logic, the schema *Prop and the rule of modus ponens are likely to be uncontroversial. The rule of uniform substitution follows directly from the instance account of informal validity: Any natural language instance of a substitution instance of a formula is also a natural language instance of the original formula, so if a substitution instance of a formula has a false natural language instance, so does the original formula. Thus if a formula is informally valid (has no false natural language instances), so does any of its substitution instances. It is not quite clear how to argue for the instances of $* K_{\text {? }}$, but such (generalized) distributivity principles are widely accepted (see also Williamson (2013, p. 98) for the case of necessity).

Claiming that Act is informally sound is more controversial. Part of the controversy comes from the philosophical discussion of $\mathbf{S 5}_{\square}$, where especially the axioms $4_{\square}$ and $5 \square$ have been criticized. Maybe the most well-known arguments against $4 \square$ are those in Salmon (1989); see also Gregory (2011) for further references. Another part that is likely to be controversial is the claim that the axioms of $P_{\text {Act }}$ involving @ are informally valid. Especially the axiom $T_{@}$ has been called into question, e.g., in Crossley \& Humberstone (1977, p. 15) and Hanson (2006). However, on the instance account of informal validity, there seems to be an easy way of arguing for it: for any sentence $S$, the truth of $\ulcorner$ If actually $S$ then $S\urcorner$ seems to be guaranteed by the semantics of "actually". So in this example, the differences in evaluating the informal validity of $T_{@}$ may be rooted in differences concerning the understanding of informal validity.

As with Act, arguing that $\mathbf{S 5} \mathbf{5}_{A}$ is informally sound is likely to be a challenging philosophical undertaking. Similar to above, it is the axioms $4_{A}$ and $5_{A}$ which are controversial. (If we use generalization for $A$ instead of $4_{A}$, see remarks on that rule below.) E.g., Humberstone (2004, p. 28) claims that apriority is suitably similar to the notions of 
demonstrability or informal provability, and refers to Burgess (1999) and others who claim that the logic of these notions is not $\mathbf{S 5}$ as it does not obey the principle 5. But to do defenders of two-dimensional semantics justice, we have to understand a priori as they do. Chalmers (2004, p. 208) describes this by saying that what is a priori is what "can be conclusively nonexperientially justified on ideal rational reflection". Without further argument, it is not clear whether on this understanding, apriority is in fact "suitably similar" to the notions of demonstrability and informal provability. One would have to show that the particular arguments against 5 concerning demonstrability or informal provability apply to apriority as well.

However, there are further worries concerning the informal validity of $4_{A}$ and $5_{A}$. Firstly, vagueness might provide counterexamples to $4_{A}$, similar to the logic of definitely. Consider a sorites series, e.g., consisting of suitably arranged collections of grains of sand. Take the numeral $n$ of the least natural number such that $\ulcorner$ It is a priori that $n$ grains of sand make a heap $\urcorner$ is true. Is then $\ulcorner$ It is a priori that ... it is a priori that $n$ grains of sand make a heap $\urcorner$ true for any number of iterations of "it is a priori that"? If not, the $4_{A}$ principle is false on the instance account of informal validity.

A second worry concerns unentertainable propositions. Since unentertainable propositions are likely not to be expressible by sentences, let's consider the account of informal validity as having a true universal generalization (keeping in mind that we have to be careful with propositional quantifiers in the present context). Assume that if $P$ is unentertainable, then so is any proposition containing $P$, e.g., the negation of $P$. Further, assume that being a priori entails being entertainable. Then if $P$ is not entertainable, $P$ is not a priori. Since the proposition that $P$ is not a priori contains $P$, it is not entertainable, and therefore not a priori. So $P$ is a counterexample to $\forall p(\neg A p \rightarrow A \neg A p)$, and so shows that $5_{A}$ is not informally valid. We can also use such propositions to argue that $C$ should not be read as conceivable, if we assume that being conceivable entails being entertainable: If $P$ is not entertainable, then $P$ is not a priori, but then the negation of $P$ is also not entertainable, so the negation of $P$ is not conceivable. Thus if $C$ is read conceivably, then $P$ is a counterexample to $\forall p(\neg A p \rightarrow C \neg p)$, which follows from the duality of $A$ and $C$.

Of course, all of this is only worrying if there are any unentertainable propositions. To consider this, note that there are different ways unentertainable could be understood. One way to understand it would be to take it to mean it is (metaphysically) possible that someone entertains $p$. Then there might well be examples of unentertainable propositions; see Fritz (2013, p. 1765) for an example. But as pointed out there, we shouldn't assume without further argument that a proposition being a priori means that it is (metaphysically) possible that someone knows it a priori, and thus there is no reason to assume that apriority implies entertainability on the present understanding of the latter. To make the objection work, we need propositions that are unentertainable in some stronger sense, such that it is clear that being a priori implies being entertainable. Are there any such propositions?

One might think that this follows from a family of intensional paradoxes going back to Prior (1961) (see Tucker \& Thomason (2011) for a recent discussion). Consider the following case. Assume for contradiction that I uniquely entertain that everything uniquely entertained by me is false. Now, either everything uniquely entertained by me is false or not everything uniquely entertained by me is false. If we assume that everything uniquely entertained by me is false, then since this is what I uniquely entertain, it follows that I uniquely entertain something true, contradicting the assumption. If we assume that not everything uniquely entertained by me is false, it follows that something is uniquely entertained by 
me and true. So since I uniquely entertain that everything uniquely entertained by me is false, everything uniquely entertained by me is false, contradicting the assumption. Thus I do not uniquely entertain that everything uniquely entertained by me is false. Hence we can show on logical grounds that there is something I do not uniquely entertain. Of course, there is nothing special about me that allows this argument to go through. So plausibly, what I do not uniquely entertain cannot be uniquely entertained by anyone. And since the argument only relied on a few basic principles of inference, it is plausible that uniquely entertaining it is not only metaphysically impossible, but impossible on broadly logical grounds. However, even if we can argue that being a priori implies being entertainable (in this very wide sense), this does not give us an arguments against $5_{A}$, since the kind of unentertainability used here does not transfer from a proposition to any proposition containing it. Furthermore, the argument does not go through if being a priori only implies being entertainable, rather than being uniquely entertainable, and we have not been given a reason to make the stronger assumption.

Besides arguing for the informal soundness of $\mathbf{K}$, Act and $\mathbf{S} \mathbf{5}_{A}$, we also have to argue for the additional principles of $P_{\mathbf{2 D}}$. Consider first the role of generalization for $A$ on the instance account of informal validity. While it doesn't seem implausible that $A \varphi$ has no false instances if $\varphi$ has no false instances, there is also no obvious positive argument. Consider now the extra axioms of $P_{2 \mathbf{D}}$. While all of them say something about the interactions between necessity and apriority, $N 1$ stands out. One difference between it and the others is that $\square 4_{A}, \square 5_{A}$ and $\square D_{A}$ are all necessitations of theorems of $\mathbf{S 5}_{A}$, in contrast to $N 1$. $\square D_{A}$ is a highly plausible principle as violating it would mean (on the instance account of informal validity) that there is a sentence $S$ such that $\ulcorner$ Possibly, it is a priori that $S$ and a priori that not $S\urcorner$ is true. It is hard to imagine how this could be true. $\square 4_{A}$ and $\square 5_{A}$ are necessitations of principles considered above, and therefore face similar difficulties as discussed there.

$N 1$ is of special interest, as it plays a role in the so-called nesting problem, which is discussed in some detail in Fritz (2013, section 4). The nesting problem originates from Soames (2005, see Argument 5 on pp. 278-279), and it is presented in Chalmers (2011, endnote 25) as the following argument, concluding $N 3$ from premises $N 1$ and $N 2$ :

$$
\begin{array}{ll}
N 1 & A p \rightarrow \square A p \\
N 2 & \square(A p \rightarrow p) \\
N 3 & A p \rightarrow \square p
\end{array}
$$

For a variant of this argument, Soames effectively argues that two-dimensional semantics is committed to the truth of the first premise, that the second premise is true, that the the conclusion is false, and that therefore, the argument refutes two-dimensional semantics. If one accepts the validity of the argument, it is natural to claim that the relevant instance of $N 1$ is in fact false, and therefore cannot be informally valid. No matter what the correct answer to the nesting problem is, it shows that $N 1$ is a controversial axiom. It also appears to capture a central aspect of the relations between necessity and apriority according to $2 \mathbf{D}$. It is therefore interesting to prove that it essentially captures this aspect in $P_{\mathbf{2 D}}$, by showing that $N 1$ cannot be deduced if it is removed from $P_{2 \mathbf{D}}$ :

DEFINITION 2.20. Let $P_{2 \mathbf{D}}^{-}$be the proof system containing the same rules and the same axioms, except for $N 1$, as $P_{2 \mathbf{D}}$.

$$
\text { Proposition 2.21. } \nvdash_{P_{2 \mathbf{D}}^{-}} N 1 \text {. }
$$


Proof. Consider the FWDE $\mathcal{F}$ given by the following diagram, with points 0 and 1 of which 0 is distinguished:

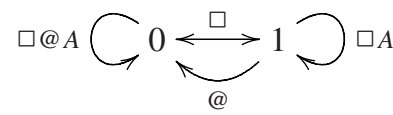

It is routine to show that the logic of $P_{\mathbf{2 D}}^{-}$is valid in $\mathcal{F}$. To see that $\mathcal{F} \nVdash N 1$, consider a valuation $V$ such that $V(p)=\{0\}$.

Overall, we have seen that a number of axioms which have to be shown to be informally valid to argue that 2D is informally sound are quite controversial. Thus arguing for the informal soundness of $\mathbf{2 D}$ will likely be philosophically demanding. The current outline is also interesting if one wants to argue against the informal soundness of 2D, since it allows us to limit our attention to a finite number of axioms (or axiom schemas) and rules among which we are guaranteed to find ones that are informally invalid, or do not preserve informal validity, if $\mathbf{2 D}$ is not informally sound.

§3. Informal Completeness. Let's consider how one could argue that 2D is informally complete, that is, that every informally valid formula is a theorem of 2D. I start by illustrating the strategy using the logic $\mathbf{S 5} \square$ for necessity in more detail. The argument for informal completeness builds on that for informal soundness, so we assume that $\mathbf{S 5}_{\square}$ is informally sound, and then ask the question whether it is also informally complete. In addition, we assume - as argued for in the informal soundness argument - that the informal validities are closed under modus ponens and uniform substitution. The basic idea of the argument is to show that any logic that contains $\mathbf{S 5}_{\square}$ and is closed under these rules is either $\mathbf{S 5}_{\square}$ itself or contains a formula that is not informally valid. With the assumptions, the informal completeness of $\mathbf{S 5}_{\square}$ follows.

To make this strategy precise, let me introduce the following terminology: An extension of a $\operatorname{logic} \Lambda$ is a set of formulas that includes $\Lambda$. Such an extension is proper if it properly includes it, i.e. if it contains a formula not in $\Lambda$. Further, for any $n \in \mathbb{N}$ and modality $\nabla$, define the following formula:

$$
\mathrm{Alt}_{n}^{\nabla}=\bigvee_{0 \leq i \leq n} \nabla\left(\left(\bigwedge_{1 \leq j \leq i} p_{j}\right) \rightarrow p_{i+1}\right)
$$

This formula is valid at a point in a frame or FWDE if and only if the point can reach at most $n$ points via the relation for $\nabla$; see Segerberg (1971, p. 52, proof of Lemma 5.3). Other formulas expressing the same condition be found in Dugundji (1940) and Gärdenfors (1973).

With these definitions, we can state the argument: Assume for contradiction that $\mathbf{S 5}_{\square}$ is not informally complete. Since by assumption, $\mathbf{S 5}_{\square}$ is informally sound and the informal validities are closed under modus ponens and uniform substitution, they form a proper quasi-normal extension of S5 $\square$. By Scroggs's theorem, proven in Scroggs (1951), every such logic is characterized by a single finite frame with a universal relation. Let $n$ be the number of elements in the frame that characterizes the informal validities. Then $\mathrm{Alt}_{n}^{\square}$ is valid on that frame and therefore informally valid. But $\mathrm{Alt}_{n}^{\square}$ is not informally valid. Contradiction, so $\mathbf{S 5}_{\square}$ is informally complete.

Why is $\mathrm{Alt}_{n}^{\square}$ not informally valid? Consider the instance account of informal validity, and the instance of $\mathrm{Alt}_{n}^{\square}$ obtained by letting every $p_{i}$ stand for $\ulcorner$ There are at least $i$ donkeys $\urcorner$. Then a disjunct of Alt ${ }_{n}^{\square}$ says that necessarily, if there are at least $i$ donkeys, then there are at least $i+1$ donkeys. But plausibly, for every natural number $i$, it is possible that 
there are exactly $i$ donkeys, so it follows that all disjuncts of this instance of Alt ${ }_{n}^{\square}$ are false, and hence that this instance of Alt $t_{n}^{\square}$ itself is false.

Scroggs's theorem was originally formulated in an algebraic setting, in which we can state it by saying that while $\mathbf{S 5}$ is not characterized by a finite matrix, all of its proper extensions are characterized by a finite matrix; this property is called pretabularity. (Matrices are algebraic structures that can be used like frames to interpret modal languages; note that there is no relation to the terminology of matrix frames.) It is not hard to see that $2 \mathbf{D}$ is not pretabular. But as we will see, pretabularity is not required to run the kind of argument just illustrated using $\mathbf{S 5}$ - we can do with local tabularity (see below).

3.1. Characterizing Extensions of 2D. To be able to construct an analogous argument for the informal completeness of $\mathbf{2 D}$, we first need some technical results that characterize the extensions of 2D in a useful way. The natural idea is to show that any proper quasinormal extension of 2D is characterized by a class of FWDEs that are somehow limited in size, which entails that it contains a formula somehow analogous to $\mathrm{Alt}_{n}^{\square}$. Note that we can limit the size of an FWDE for 2D in two ways; on the one hand, we can limit the number of points accessible via the relation for $A$, and on the other hand, we can limit the number of points accessible via the relation for $\square$. It is therefore not surprising that the analogous formulas should contain instances of $\mathrm{Alt}_{n}^{\nabla}$ for both of the modalities $A$ and $\square$. As it turns out, we can use the formulas of the form $\mathrm{Alt}_{m}^{A} \vee C \mathrm{Alt}_{n}^{\square}$ for natural numbers $m$ and $n$, as we can show that any proper quasi-normal extension of $\mathbf{2 D}$ contains one of them. This is the characterization result we will prove in this section.

To do so, we start by proving a normal form theorem, which shows that every formula can equivalently be written in a certain syntactically simple form. From this, we can derive the fact that all quasi-normal extensions of 2D have the finite model property, which we can use to show that every such extension is characterized by a class of finite FWDEs based on Restall frames. From this, the characterization result can be derived.

For the normal form theorem, we prove that any formula $\varphi$ is $\mathbf{2 D}$-equivalent to a formula $\psi$ of a certain syntactically simple form, where $\varphi$ and $\psi$ are 2D-equivalent if $\varphi \leftrightarrow \psi$ is a theorem of 2D. This is an extension of a familiar result on the logic S5, for which we can prove that every formula is equivalent to a formula that contains no nested modal operators. More specifically, let a formula be in $\mathbf{S 5}_{\square-\mathrm{CNF}}$ (CNF stands for conjunctive normal form) if it is a finite conjunction of finite disjunctions of formulas of the form $\square \varphi, \diamond \varphi$ or $\varphi$, where $\varphi$ contains no modal operators. We can then prove that every formula (in the language containing only the modality $\square$ ) is $\mathbf{S 5}_{\square}$-equivalent to one in $\mathbf{S 5}_{\square}$-CNF; see Hughes \& Cresswell (1996, p. 101). The analog to containing no nested operators in the context of 2D is to contain only $\square$ and $A$, and these only unnestedly or with $\square$ nested in $A$. More precisely, we can define the following normal form:

DEFINITION 3.1. A formula is in 2D-CNF if it is a finite conjunction of finite disjunctions



\section{THEOREM 3.2. Every formula is 2D-equivalent to one in 2D-CNF.}

Proof. Define a formula to be in $\mathbf{2 D g}-\mathrm{CNF}$ if it is a finite conjunction of finite disjunctions of formulas of the form $A \varphi, C \varphi$ or $\psi$, where $\varphi$ is in $\mathbf{S 5}_{\square-C N F}$ and $\psi$ is in $\mathbf{S 5} \square-C N F$ or of the form @ $\chi$, where $\chi$ contains no modal operators. We can prove that every formulas is $\mathbf{2 D g}$-equivalent to one in $\mathbf{2 D g}$-CNF by an induction on the complexity of formulas analogously to the case of $\mathbf{S 5} \square-\mathrm{CNF}$. Any formula in $\mathbf{2 D g}$-CNF contains @ only unnestedly. 
The claim follows, as removing any such occurrence of @ produces a 2D-equivalent formula in 2D-CNF.

Similar to the well-known result of Bull (1966) that all normal extensions of $\mathbf{S 4 . 3}$ have the finite model property, every quasi-normal extension of $\mathbf{2 D}$ has the finite model property, which means that it is characterized by a class of finite models. We can show this by noting that the normal form theorem just proven entails that 2D is locally tabular, which means that any set of formulas that are pairwise nonequivalent in 2D and jointly contain only finitely many proposition letters is finite itself:

\section{LEMMA 3.3. Every quasi-normal extension of 2D has the finite model property.}

Proof. By Theorem 3.2, 2D is locally tabular. This property is preserved in any quasinormal extension of $\mathbf{2 D}$, so the claim to be proven follows from the fact that every locally tabular quasi-normal modal logic has the finite model property. (See Humberstone (2011, p. 228, Observation 2.13.5 (ii)); the claim can also be proven using a filtration argument along the lines of Segerberg (1971, p. 129, Theorem 6.1)).

LEMMA 3.4. Every quasi-normal extension of 2D is characterized by a class of finite FWDEs based on Restall frames.

Proof. Let $\Lambda$ be a quasi-normal extension of 2D. The well-known result that a logic with the finite model property has the finite frame property, see, e.g., Blackburn et al. (2001, Theorem 3.28), is straightforwardly extended to structures with distinguished elements. Thus it follows from Lemma 3.3 that $\Lambda$ is weakly complete with respect to the class of finite FWDEs it defines. Since 2Dg $\subseteq$ 2D is normal, it follows from Lemma 2.6 that any FWDE generated by a distinguished point of such an FWDE is based on a Restall frame. Thus the class of such FWDEs witnesses the claim of this lemma.

With this, we can prove the theorem needed for the informal completeness argument:

THEOREM 3.5. If $\Lambda$ is a proper quasi-normal extension of $\mathbf{2 D}$, then there are $m, n \in \mathbb{N}$ such that $\vdash_{\Lambda} \operatorname{Alt}_{m}^{A} \vee C \mathrm{Alt}_{n}^{\square}$.

Proof. Consider any quasi-normal $\Lambda \supseteq$ 2D such that for no $m, n \in \mathbb{N}, \vdash_{\Lambda} \operatorname{Alt}_{m}^{A} \vee C \mathrm{Alt}_{n}^{\square}$. We prove that $\Lambda=\mathbf{2 D}$. Let $\varphi \notin \mathbf{2 D}$. By Lemma 3.4, there is a finite FWDE $\mathcal{F}$ based on a Restall frame such that $\mathcal{F}, V, w \nVdash \varphi$ for some valuation $V$ and distinguished point $w$. Let $m$ be the number of equivalence classes of points in $\mathcal{F}$ under the $\square$-relation and $n$ the cardinality of the largest such equivalence class. Since $\nvdash_{\Lambda} \mathrm{Alt}_{m}^{A} \vee C \mathrm{Alt}_{n}^{\square}$, it follows from Lemma 3.4 that there is an FWDE $\mathcal{F}^{\prime}$ based on a Restall frame such that $\mathcal{F}^{\prime} \Vdash \Lambda$ and $\mathcal{F}^{\prime} \nVdash \mathrm{Alt}_{m}^{A} \vee C \mathrm{Alt}_{n}^{\square}$. Thus $\mathcal{F}^{\prime}$ contains at least $m$ equivalence classes of points under the $\square$-relation and every such class has cardinality $\geq n$. So one can choose a valuation $V^{\prime}$ and distinguished point $w^{\prime}$ such that there is a bounded morphism (see Blackburn et al. (2001, pp. 57-63)) from $\mathcal{F}^{\prime}, V^{\prime}$ to $\mathcal{F}, V$ mapping $w^{\prime}$ to $w$. Since truth is invariant under bounded morphisms, $\mathcal{F}^{\prime}, V^{\prime}, w^{\prime} \nVdash \varphi$, and therefore $\varphi \notin \Lambda$. Hence $\Lambda=\mathbf{2 D}$.

One might wonder why one can't obtain proper extensions of 2D by strengthening the interaction principles between modalities without including formulas of the form $\operatorname{Alt}_{m}^{A} \vee$ $C \mathrm{Alt}_{n}^{\square}$. E.g., one might wonder why the smallest quasi-normal extension of $\mathbf{2 D}$ containing $\square p \rightarrow A p$ isn't a counterexample to Theorem 3.5. This seems puzzling if we only focus on $\square$ and $A$, but using @, the question is easily answered: By uniform substitution, the proposed extension contains $\square @ p \rightarrow A @ p$, and so with the 2D-theorems $p \rightarrow \square @ p$ and $A @ p \rightarrow A p$, it contains $p \rightarrow A p$, from which we obtain $\mathrm{Alt}_{1}^{A}$ by a routine derivation. 
In general, this suggests that although Theorem 3.5 characterizes the extensions of 2D using formulas which do not include the actuality operator, the interactions of the actuality operator with the other operators in $\mathbf{2 D}$ are essential to it.

Before moving on to the outline of the informal completeness argument, let me note that we get two further properties of $\mathbf{2 D}$ as easy corollaries from the work done in this section, which are worth pointing out. Firstly, the actuality operator is redundant in $2 \mathbf{D}$ in the following sense:

COROLlARY 3.6. For every formula, there is a 2D-equivalent formula not containing @.

Proof. Immediate from Theorem 3.2, since no formula in 2D-CNF contains an occurrence of @.

The analogous result for Act was already shown in both Crossley \& Humberstone (1977) and Hazen (1978). Hazen et al. (2013) extend it to logics of necessity and actuality based on logics of necessity other than $\mathbf{S 5}_{\square}$. For a range of tense logics with an operator representing now, the analogous result was already proven in Kamp (1971, p. 251).

Secondly, we can prove:

\section{Corollary 3.7. 2D is decidable.}

Proof. We have seen that 2D is finitely axiomatizable, and by Lemma 3.3, it has the finite model property. Decidability follows from these two properties; see, e.g., the proof of Blackburn et al. (2001, Theorem 6.15).

3.2. Outline of an Informal Completeness Argument. As in the case of $\mathbf{S 5}_{\square}$, we can now outline an argument for the informal completeness of $\mathbf{2 D}$ on the assumption that $2 \mathbf{D}$ is informally sound and that modus ponens and uniform substitution preserve informal validity. By Theorem 3.5, it follows from these assumptions that if $\mathbf{2 D}$ is informally incomplete, then there are natural numbers $m$ and $n$ such that $\operatorname{Alt}_{m}^{A} \vee C$ Alt $_{n}^{\square}$ is informally valid. So it only remains to argue that no such formula is informally valid.

3.3. Arguing for the Premise. How controversial is it to claim that for any natural numbers $m$ and $n, \mathrm{Alt}_{m}^{A} \vee C \mathrm{Alt}_{n}^{\square}$ is informally invalid? Let me use the instance account of informal validity again to consider this. As in the case of $\mathbf{S 5}_{\square}$, we can argue for the claim by considering arbitrary natural numbers $m$ and $n$, and the instance of the formula obtained by letting every $p_{i}$ stand for $\ulcorner$ There are at least $i$ donkeys $\urcorner$. We have to show that neither of the disjuncts of this instance is true. The first says that for some $i \leq m$, it is a priori that if there are at least $i$ donkeys, then there are at least $i+1$ donkeys. But it is plausible that for any natural number $i$, it is not a priori that that there are not exactly $i$ donkeys. Thus the relevant instance of $\mathrm{Alt}_{m}^{A}$ is false. Now consider the second disjunct. It says that it is not a priori that there is no natural number $i \leq n$ such that necessarily, if there are at least $i$ donkeys, then there are at least $i+1$ donkeys. But we have argued before that for every natural number $i$, it is possible that there are exactly $i$ donkeys. So in particular, this is the case for all $i \leq n$. This reasoning was a priori, so it is a priori that there is no $i \leq n$ such that necessarily, if there are at least $i$ donkeys, then there are at least $i+1$ donkeys. This means that the relevant instance of $C \mathrm{Alt}_{n}^{\square}$ is false. So $\mathrm{Alt}_{m}^{A} \vee C \mathrm{Alt}_{n}^{\square}$ has a false instance, and is therefore not informally valid.

So at least on the instance account of informal validity, it seems quite plausible that the relevant formulas are in fact not informally valid. Thus the informal completeness 
of 2D is a relatively uncontroversial consequence of its informal soundness; the premises in the argument for the informal soundness of $\mathbf{2 D}$ were clearly much more controversial.

§4. A Variant Logic. I have now concluded an outline of an argument for the correctness of 2D. Its most contentious premise is probably the informal validity of $N 1=A p \rightarrow$ $\square A p$, which requires us to reject $N 2=\square(A p \rightarrow p)$, given the plausible background assumptions that the correct logic is quasi-normal and does not contain $N 3=A p \rightarrow$ $\square p$. Most philosophers not antecedently committed to two-dimensional semantics will probably take this to be a compelling argument against the correctness of 2D. This raises the question whether there are any plausible alternatives to $2 \mathrm{D}$ which validate $N 2$ but reject $N 1$. In this section, I tentatively explore one such logic. I first specify a logic using a class of FWDEs closely related to matrix FWDEs, and then show that this logic is in a certain sense a minimal variant of $\mathbf{2 D}$ containing $N 2$ instead of $N 1$.

As in the case of matrix FWDEs, truth is relativized to two indices, a metaphysical possibility and an epistemic possibility. We keep the interpretation of necessity as truth in all metaphysical possibilities, keeping the epistemic possibility fixed. But in contrast to matrix FWDEs, we now interpret apriority exactly analogously, i.e., as truth in all epistemic possibilities, keeping the metaphysical possibility fixed. To interpret actuality, we distinguish one metaphysical possibility as the one which is realized, and interpret actuality as truth in the distinguished metaphysical possibility, keeping the epistemic possibility fixed. As in the case of matrix FWDEs, formal models are defined with the simplifying assumption that the metaphysical possibilities are the epistemic possibilities; again, this will make no difference to the logic considered here.

Definition 4.1. Let $\mathfrak{F}=\left\langle W, R_{\square}, R_{@}, R_{A}\right\rangle$ be a product matrix frame if there is a set $S$ and $s \in S$ such that $W=S \times S$ and

- $\langle x, y\rangle R_{\square}\left\langle x^{\prime}, y^{\prime}\right\rangle$ iff $y=y^{\prime}$

- $\langle x, y\rangle R_{@}\left\langle x^{\prime}, y^{\prime}\right\rangle$ iff $y=y^{\prime}$ and $x^{\prime}=s$

- $\langle x, y\rangle R_{A}\left\langle x^{\prime}, y^{\prime}\right\rangle$ iff $x=x^{\prime}$

Let $\mathcal{F}=\left\langle W, R_{\square}, R_{@}, R_{A}, D\right\rangle$ be a product matrix FWDE if $\left\langle W, R_{\square}, R_{@}, R_{A}\right\rangle$ is a product matrix frame (with $S$ and $s$ as above) and $D=\{\langle s, y\rangle: y \in S\}$.

Let $\mathrm{P}$ be the class of product matrix frames and $\mathrm{PD}$ the class of product matrix FWDEs.

The term "product matrix frames" is motivated by the fact that in the terminology of product logics (see, e.g, Gabbay et al. (2003, chapter 5)), the result of removing the accessibility relation for @ from a product matrix frame is called the "product" of a frame with a universal relation with itself. In the following, we investigate the logic of PD, first providing an axiomatization, and then briefly considering the prospects for outlining a correctness argument analogous to the above correctness argument for 2D.

4.1. Axioms for the logic characterized by PD. Our axiomatization of the logic of PD follows the axiomatization of the logic of MD in section 2.1.; we first axiomatize the logic of the class of frames $P$ and then derive the logic of PD. To axiomatize the logic of $\mathrm{P}$, we start with the axioms for Actg, to which we can now add the axioms of $\mathbf{S 5}_{A}$, as in product matrix frames, the accessibility relation for $A$ is an equivalence relation. From the literature on product logics, we know that important features of the interaction of $\square$ and $A$ are captured by the three formulas $\operatorname{com}_{\square A}(\square A p \rightarrow A \square p), \operatorname{com}_{A \square}(A \square p \rightarrow \square A p)$ and $c h r_{\square A}(\diamond A p \rightarrow A \diamond p)$. It turns out that in the presence of the other axioms, it suffices to 
add $\operatorname{com}_{A \square}$ as an axiom. Finally, we add an axiom corresponding to $I 4$ in the definition of 2Dg, namely $I 5=@ A(@ p \rightarrow p)$.

DEFINITION 4.2. Let $\mathbf{S 5}^{\mathbf{2}} @ \mathbf{g}$ be the normal modal logic axiomatized by the following formulas:

$$
\begin{array}{llll}
T \square & \square p \rightarrow p & I 2 & @ p \rightarrow \square @ p \\
5 \square & \diamond p \rightarrow \square \diamond p & T_{A} & A p \rightarrow p \\
D_{@} & @ p \rightarrow \neg @ \neg & 5_{A} & C p \rightarrow A C p \\
D_{c} @ & \neg @ \neg \rightarrow @ p & c o m_{A} \square & A \square p \rightarrow \square A p \\
I 1 & \square p \rightarrow @ p & I 5 & @ A(@ p \rightarrow p)
\end{array}
$$

Define $\mathbf{S 5}^{2} @$ such that $\vdash_{\mathbf{S 5}^{2} @} \varphi$ iff $\vdash_{\mathbf{S 5}^{2} @ \mathbf{g}} @ \varphi$.

As in the case of the axioms of $\mathbf{2 D g}$, all of these axioms are Sahlqvist formulas, and we can therefore straightforwardly compute their local frame-correspondents and conclude that $\mathbf{S 5}^{\mathbf{2}} @ \mathbf{g}$ is sound and strongly complete with respect to the class of frames it defines. The correspondents of the new formulas are as follows:

$$
\begin{array}{lll}
\operatorname{com}_{A \square} & A \square p \rightarrow \square A p & \forall v u\left(\left(w R_{\square} v \wedge v R_{A} u\right) \rightarrow \exists x\left(w R_{A} x \wedge x R_{\square} u\right)\right) \\
I 5 & @ A(@ p \rightarrow p) & \forall v u\left(\left(w R_{@} v \wedge v R_{A} u\right) \rightarrow u R_{@} u\right)
\end{array}
$$

\section{THEOREM 4.3. $\mathbf{S 5}^{\mathbf{2}} @ \mathbf{g}$ is sound and strongly complete with respect to $\mathrm{Fr}_{\mathbf{S 5}} \mathbf{2}_{\mathbf{g}}$.}

Proof. By Sahlqvist's completeness theorem.

As in the case of matrix frames, we use this to derive a completeness theorem with respect to the class of product matrix frames, but without proving that the point-generated subframes of $\mathbf{S 5}^{\mathbf{2}} @ \mathbf{g}$ frames are the bounded morphic images of product matrix frames. We still prove completeness through an intermediate class of frames, but the structural connections will be less tight. The following is partly based on an existing proof strategy for proving completeness for product logics using countable frames and bounded morphic images (see, e.g, Gabbay et al. (2003, section 5.1)). We start by defining the intermediate class of frames.

DEFINITION 4.4. A P matrix frame is a frame $\mathfrak{F}=\left\langle W, R_{\square}, R_{@}, R_{A}\right\rangle$ such that

- $R_{\square}$ and $R_{A}$ are equivalence relations,

- for all $w, v \in W,[w]_{R_{\square}} \cap[v]_{R_{A}} \neq \emptyset$,

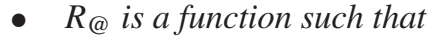

(i) if $w R_{@ v} v$ then $w R_{\square} v$ and

(ii) $R_{@}$ maps any two $R_{\square}$-related points to the same point, and

- $\operatorname{im}\left(R_{@}\right) \in W / R_{A}$.

Here, $X / E$ is the set of equivalence relations of elements of a set $X$ under an equivalence relation $E$ on $X$. Note that for every point $w$ in a P matrix frame, $\operatorname{im}\left(R_{@}\right) \cap[w]_{R_{\square}}$ is a singleton.

LEMMA 4.5. Every point-generated subframe of an $\mathbf{S 5}^{\mathbf{2}} @ \mathbf{g}$ frame is a P matrix frame.

Proof. Consider any $\mathbf{S 5}^{2} @ \mathbf{g}$ frame $\mathfrak{F}=\left\langle W, R_{\square}, R_{@}, R_{A}\right\rangle$ and $w \in W$. Let $\mathfrak{F}_{w}=$ $\left\langle W^{\prime}, R_{\square}^{\prime}, R_{@}^{\prime}, R_{A}^{\prime}\right\rangle$ be the subframe of $\mathfrak{F}$ generated by $w$. Note that $\mathfrak{F}_{w}$ is an $\mathbf{S 5}^{\mathbf{2}} @ \mathbf{g}$ frame as well. Using $T_{\square}, 5_{\square}, T_{A}$ and $5_{A}$, it is routine to show that $R_{\square}^{\prime}$ and $R_{A}^{\prime}$ are equivalence 
relations; similarly, it is routine to show using $D_{@}$ and $D_{c} @$ that $R_{@}^{\prime}$ is a function, and with $I 1$ and $I 2$ that it satisfies conditions (i) and (ii). To establish the remaining two conditions, we first prove the following auxiliary claim:

Claim 1: $W^{\prime}=Y$, where $Y=R_{\square}\left[R_{A}[\{w\}]\right]$. Clearly $Y \subseteq W^{\prime}$. To prove that $W^{\prime} \subseteq Y$, we first show that $w \in Y$, and then that $Y$ is closed under each relation; i.e., $R_{\nabla}[Y] \subseteq Y$ for each modality $\nabla . w \in Y$ follows from the fact that both $R_{A}$ and $R_{\square}$ are reflexive. $R_{\square}[Y] \subseteq Y$ follows from the transitivity of $R_{\square}$. For $R_{@}$, assume that $w R_{A} v R_{\square} u R_{@ x} x$. By $I 2, v R_{@} x$, so by $I 1, v R_{\square} x$. Hence $R_{@}[Y] \subseteq Y$. For $R_{A}$, assume that $w R_{A} v R_{\square} u R_{A} x$. By $\operatorname{com}_{A \square}$, there is a $y \in W$ such that $w R_{A} v R_{A} y R_{\square} x$, and so by the transitivity of $R_{A}$, $w R_{A} y R_{\square} x$. Hence $R_{A}[Y] \subseteq Y . \checkmark$

Claim 2: For all $v, u \in W^{\prime},[v]_{R_{\square}^{\prime}} \cap[u]_{R_{A}^{\prime}} \neq \emptyset$. Consider any $v, u \in W^{\prime}$. By claim 1, there are $x, y \in W^{\prime}$ such that $v R_{\square}^{\prime} x R_{A}^{\prime} w R_{A}^{\prime} y R_{\square}^{\prime} u$. By transitivity of $R_{A}^{\prime}, x R_{A}^{\prime} y$, so by $\operatorname{com}_{A \square}$, there is a $z \in W^{\prime}$ such that $u R_{A}^{\prime} z R_{\square}^{\prime} x$. Therefore $z R_{\square}^{\prime} v$, and so $z \in[v]_{R_{\square}^{\prime}} \cap$ $[u]_{R_{A}^{\prime} \cdot \checkmark}$

Claim 3: $\operatorname{im}\left(R_{@}^{\prime}\right) \in W^{\prime} / R_{A}^{\prime}$. We show that $\operatorname{im}\left(R_{@}^{\prime}\right)=\left[w^{\prime}\right]_{R_{A}^{\prime}}$, where $w^{\prime}=R_{@}^{\prime}(w)$. Consider first any $v^{\prime} \in \operatorname{im}\left(R_{@}^{\prime}\right)$. Then there is a $v \in W^{\prime}$ such that $R_{@}^{\prime}(v)=v^{\prime}$. By claim 2, there is a $u \in W^{\prime}$ such that $w^{\prime} R_{A}^{\prime} u R_{\square}^{\prime} v$, and therefore $u R_{@}^{\prime} v^{\prime}$. It follows with $I 5$ that $u R_{@}^{\prime} u$, so $u=v^{\prime}$, and therefore $v^{\prime} \in\left[w^{\prime}\right]_{R_{A}^{\prime}}$. Consider now any $v \in\left[w^{\prime}\right]_{R_{A}^{\prime}}$. Then $w R_{@}^{\prime} w^{\prime} R_{A}^{\prime} v$, so by $I 5, v R_{@}^{\prime} v$, and therefore $v \in \operatorname{im}\left(R_{@}^{\prime}\right) . \checkmark$

LEMMA 4.6. Every countable P matrix frame is a bounded morphic image of a product matrix frame.

Proof. Let $\mathfrak{F}=\left\langle W, R_{\square}, R_{@}, R_{A}\right\rangle$ be a countable P matrix frame (i.e., $|W| \leq \aleph_{0}$ ). We take $\mathbb{Z}$ to be the set of integers. Let $\alpha$ be a surjection from $\mathbb{Z}$ to $W / R_{A}$ such that $\alpha(0)=\operatorname{im}\left(R_{@}\right)$ and $\beta$ a surjection from $\mathbb{Z}$ to $W / R_{\square}$. For each $i, j \in \mathbb{Z}$, let $\gamma_{i j}$ be a surjection from $\mathbb{Z}$ to $\alpha(i) \cap \beta(j)$. Such functions exist for cardinality reasons. Let $\mathfrak{F}^{\prime}$ be the product matrix frame based on the set $\mathbb{Z} \times \mathbb{Z}$, with $\langle 0,0\rangle$ being the special element used in the definition of the accessibility relation for @. Note that the set of points of $\mathfrak{F}^{\prime}$ is $W^{\prime}=(\mathbb{Z} \times \mathbb{Z}) \times(\mathbb{Z} \times \mathbb{Z})$. We define a function $f: W^{\prime} \rightarrow W$ such that for all $i, j, k, l \in \mathbb{Z}$ :

$$
f(\langle\langle i, j\rangle,\langle k, l\rangle\rangle)=\gamma_{i k}(j+l)
$$

It is routine to check that $f$ is a surjective bounded morphism from $\mathfrak{F}^{\prime}$ to $\mathfrak{F}$.

\section{THEOREM 4.7. $\mathbf{S 5}^{2} @ \mathbf{g}$ is sound and strongly complete with respect to $\mathrm{P}$.}

Proof. It is routine to show that all axioms of $\mathbf{S 5}^{\mathbf{2}} @ \mathbf{g}$ are valid on product matrix frames, and thus that $\mathbf{S 5}^{\mathbf{2}} @ \mathbf{g}$ is sound with respect to the class of product matrix frames. For strong completeness, consider any $\mathbf{S 5}^{\mathbf{2}} @ \mathbf{g}$-consistent set of formulas $\Gamma$. By Theorem 4.3, $\Gamma$ is satisfiable on a frame validating $\mathbf{S 5}^{\mathbf{2}} @ \mathbf{g}$. Adapting a standard argument (see, e.g., Gabbay et al. (2003, p. 22, Theorem 1.6)), we can show that this frame can be assumed to be countable: The axioms of $\mathbf{S 5}^{\mathbf{2}} @ \mathbf{g}$ express first-order frame conditions, so using the standard translation and the downward Löwenheim-Skolem theorem for first-order logic, we can show that $\Gamma$ is satisfiable at some point $w$ of a countable $\mathbf{S 5}^{\mathbf{2}} @ \mathbf{g}$ frame $\mathfrak{F}$. By Lemma $4.5, \mathfrak{F}_{w}$ is a P matrix frame. Since $\mathfrak{F}$ is countable, so is $\mathfrak{F}_{w}$, and thus by Lemma 4.6, it is a bounded morphic image of a product matrix frame. Using standard arguments, we can conclude that $\Gamma$ is satisfiable on such a product matrix frame, and so that $\mathbf{S 5}^{\mathbf{2}} @ \mathbf{g}$ is strongly complete with respect to the class of product matrix frames. 
As before, we can turn this completeness result on frames into a completeness result on FWDEs:

THEOREM 4.8. S5²@ is sound and strongly complete with respect to PD.

Proof. Exactly analogously to the proof of Theorem 2.11.

4.2. $\mathrm{S5}^{2} @$ and Informal Soundness. As in the case of 2D, the syntactic characterization of $\mathbf{S 5}^{\mathbf{2}} @$ obtained in the last section is not suitable for an inductive argument for informal completeness. But again, we can use this characterization to define a more useful proof system:

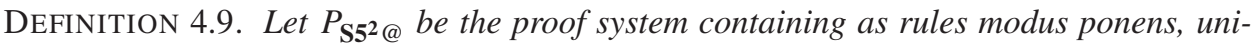
form substitution, and generalization for $A$, and as axioms the theorems of $\mathbf{K}$, Act and $\mathbf{S 5}_{A}$, as well as the following formulas:

$$
\begin{array}{ll}
\text { N2 } & \square(A p \rightarrow p) \\
\square 5_{A} & \square(C p \rightarrow A C p) \\
\square \text { com }_{A \square} & \square(A \square p \rightarrow \square A p)
\end{array}
$$

THEOREM 4.10. The logic of $P_{\mathbf{S 5}^{2} @}$ is $\mathbf{S 5}^{2} @$.

Proof. Analogous to the proof of Theorem 2.15. The only interesting differences occur in the induction on the construction of $\mathbf{S 5}^{2} @ \mathbf{g}$ showing that $\varphi \in \mathbf{S 5}^{2} @ \mathbf{g}$ implies $\vdash_{P_{\mathbf{S 5}^{2} @}} \square \varphi$. First, to prove that $\vdash_{P_{\mathbf{S 5}^{2} @}} \square I 5$, note that $\vdash_{P_{\mathbf{S 5}} \mathbf{2} @} @ p \rightarrow p$, and so $\vdash_{P_{\mathbf{S 5}} \mathbf{2} @}$ $A(@ p \rightarrow p)$. Also $\vdash_{\mathrm{S}^{2} @} @ \rightarrow \square @ p$, so by modus ponens and uniform substitution, $\vdash_{P_{\mathbf{S 5}^{2}} @} \square @ A(@ p \rightarrow p)$. Second, for generalization for $A$, we now use $\square c c_{A} \square$.

With this, it is clear how to construct an inductive informal soundness argument. Most of its premises are the same as in the argument for the informal soundness of 2D; what is new are the axioms $N 2$ and $\square \operatorname{com}_{A \square}$. That we should have to argue for the informal validity of $N 2$ was to be expected; after all, including it instead of $N 1$ motivated our consideration of $\mathbf{S 5}^{2} @$.

On their own, the three interaction principles $N 2, \square 5_{A}$ and $\square$ com $_{A \square}$ seem plausible enough; the latter two are also theorems of 2D. But it turns out that accepting them together leads to an odd picture of apriority in counterfactual situations. Take as a premise $p \wedge \neg \square p$. Using $N 2$, we can argue that in $\mathbf{S 5}^{\mathbf{2}} @$, we obtain as a consequence $\diamond \neg A(@ p \rightarrow p)$. Since it contains $\square 5_{A}, \mathbf{S 5}^{2} @$ also contains $\square(\neg A(@ p \rightarrow p) \rightarrow A \neg A(@ p \rightarrow p))$; similarly, using the fact that it contains $\square$ com $_{A \square}$, we can show that $\mathbf{S 5} 5^{2} @$ contains $\square A @(@ p \rightarrow p)$. Thus we get the following as a theorem of $\mathbf{S 5}^{2} @:(p \wedge \neg \square p) \rightarrow \diamond(\neg A(@ p \rightarrow p) \wedge$ $A \neg A(@ p \rightarrow p) \wedge A @(@ p \rightarrow p))$. On the instance account of validity, this means that, e.g., the English sentence obtained from this formula by interpreting $p$ as "it is raining" is true. But as far as I can form judgments about these claims, this seems a very odd prediction - both the second and third conjunct in the scope of $\diamond$ seem to be in tension with the first conjunct. Of course, this is not to say that the prediction can't reasonably be accepted or made sense of. Rather, this example is intended to illustrate that by rejecting $N 1$ in favor of $N 2$, principles like $\square 5_{A}$ and $\square \operatorname{com}_{A \square}$ can become much more contentious than they were previously.

Another interesting application of $P_{\mathbf{S 5}^{2} @}$ is that we can use it to prove that in a certain sense, 2D and $\mathbf{S 5}^{2} @$ differ minimally, given their disagreement concerning $N 1$ and $N 2$. This is made precise in the following theorem: 
THEOREM 4.11. Let $\Lambda$ be the intersection of $\mathbf{2 D}$ and $\mathbf{S 5}^{\mathbf{2}} @$ (i.e., the strongest quasinormal modal logic contained in both 2D and $\mathbf{S 5}^{\mathbf{2}} @$ ).

2D is the smallest quasi-normal modal logic closed under generalization for A which contains $\Lambda$ and $N 1$.

$\mathbf{S 5}^{2} @$ is the smallest quasi-normal modal logic closed under generalization for A which contains $\Lambda$ and $N 2$.

Proof. Let $P$ be the proof system containing as rules modus ponens, uniform substitution, and generalization for $A$, and as axioms the theorems of $\mathbf{K}$, Act and $\mathbf{S} \mathbf{5}_{A}$, as well as $\square 4_{A}, \square 5_{A}, \square D_{A}$ and $\square \operatorname{com}_{A} \square$. It is routine to show that the logic of $P$ is contained in both $\mathbf{2 D}$ and $\mathbf{S 5}^{\mathbf{2}} @$, and so contained in $\Lambda$. Similarly, it is routine to show that adding $N 1$ or $N 2$ as an axiom of $P$ gives us a proof system whose logic is $\mathbf{2 D}$ or $\mathbf{S 5}^{\mathbf{2}} @$, respectively, from which the claim follows.

This shows that $\mathbf{S 5}^{\mathbf{2}} @$ is a natural candidate for someone attracted to $\mathbf{2 D}$ apart from its stance on the nesting problem. However, it should be noted that the previous theorem does not obviously entail that $\mathbf{S 5}^{\mathbf{2}} @$ is the only logic which differs minimally from $\mathbf{2 D}$ in this way. It is also interesting to note that $\Lambda$ itself is not a plausible logic of the three connective, even for someone who wants to reject both $N 1$ and $N 2$, since $\Lambda$ contains their disjunction $N 1 \vee N 2$; it seems hard to argue for the informal validity of the disjunction other than by arguing for one of the disjuncts. Relatedly, $\Lambda$ contains the disjunctions of instances of $N 1$ and $N 2$ using different proposition letters, e.g., $(A p \rightarrow \square A p) \vee \square(A q \rightarrow q)$, which shows that it is not Halldén-complete, a property (Williamson, 2013, pp. 97) argues to be satisfied by any informally correct logic (see also Kripke (1965) for a related formal result).

4.3. $\mathrm{S5}^{2} @$ and Informal Completeness. To outline an argument for the informal completeness of $\mathbf{S 5}^{\mathbf{2}} @$ along the lines of the above argument for the informal completeness of 2D, we would have to characterize the extensions $\mathbf{S 5}^{\mathbf{2}} @$ in a way which allows us to argue that all of its proper extensions contain some informally invalid formula. In particular, having established Lemma 4.5 , it is natural to conjecture that we can use a similar characterization as in the case of $\mathbf{2 D}$, namely that every proper quasi-normal extensions of $\mathbf{S 5}^{\mathbf{2}}$ @ contains $\mathrm{Alt}_{m}^{A} \vee \mathrm{Alt}_{n}^{\square}$ for some natural numbers $m$ and $n$.

Unfortunately, the proof strategy used above for characterizing the extensions of 2D cannot be applied to $\mathbf{S 5}^{\mathbf{2}} @$, since $\mathbf{S 5}^{\mathbf{2}} @$ is not locally tabular. That $\mathbf{S 5}^{\mathbf{2}} @$ is not locally tabular can be established using the formulas used in the proof of Gabbay \& Shehtman (1998, p. 136, Proposition 15.8), which shows that the closely related product logic $\mathbf{S 5}^{2}$ is not locally tabular. However, we might be able to establish the analog to the central lemma that every quasi-normal extension of $\mathbf{2 D}$ has the finite model property via a different route than by local tabularity. In particular, we have reason to hope that all extensions of $\mathbf{S 5}^{\mathbf{2}} @$ do in fact have the finite model property, since all normal extensions of $\mathbf{S 5}^{2}$ have the finite model property; see the reference to Bezhanishvili (2002) in Bezhanishvili \& Marx (2003, p. 367).

\$5. Implications for Two-Dimensional Semantics. The correctness argument for 2D outlined in this paper indicates a way of arguing that 2D is the correct logic of necessity, actuality and apriority which is independent of two-dimensional semantics. But the results established here are also relevant for assessing two-dimensional semantics. If one can support the premises of the argument for the correctness of 2D, one can show that the logic of necessity, actuality and apriority according to two-dimensional semantics is correct. 
This would constitute an indirect argument in favor of two-dimensional semantics: if it gets the logic of necessity, actuality and apriority right, it gets essential aspects of what it is supposed to describe right. The considerations on how to argue for informal soundness and completeness also highlight controversial principles to which two-dimensional semantics is committed, and thereby indicate ways of using 2D to argue against two-dimensional semantics, by arguing that it incorrectly commits us to the informal validity of certain formulas.

These ways of using the formal logic 2D to argue for or against two-dimensional semantics must be qualified in an important way. As noted in Fritz (2013, p. 1762), the informal accounts of two-dimensional semantics given in the literature can be cashed out formally in different ways. Therefore, there are a number of logics that can be claimed to formalize two-dimensional semantics, of which 2D is only one natural candidate. So, e.g., if a convincing argument is presented that shows that some theorem of 2D is informally invalid, a proponent of two-dimensional semantics can reply that this is a result of 2D not capturing the ideas of two-dimensional semantics faithfully. However, in this case, it is the burden of the two-dimensionalist to spell out what is wrong with $\mathbf{2 D}$, and what the correct logic of necessity, actuality and apriority according to two-dimensional semantics is. Although the initial argument would then not lead to the desired conclusion of refuting two-dimensional semantics, it would still give rise to a debate leading to a valuable clarification of two-dimensional semantics. An example of a tentative development in this direction can already be found in the discussion of the nesting problem in (Chalmers, 2011, endnote 25) and Chalmers \& Rabern (forthcoming).

I expect that when asked about it, most philosophers would judge it to be more plausible that $N 2$ is informally valid than that $N 1$ is informally valid, and therefore that it is more plausible that $\mathbf{S 5}^{\mathbf{2}} @$ is correct than that $\mathbf{2 D}$ is correct. Proponents of two-dimensional semantics might therefore wonder whether the class of product matrix FWDEs could be construed as a plausible formalization of two-dimensional semantics as well. They might note that product matrix FWDEs are also two-dimensional in the sense that they evaluate formulas relative to tuples, representing a metaphysical and an epistemic possibility. As I will now show, this is only an option for a version of two-dimensional semantics which is not committed to the so-called "conceivability/possibility link", which roughly says that in certain cases, what is conceivable is also possible. At least in its initial development, this link formed a central component of two-dimensional semantics, as it plays a key role in the argument for the claim that conscious experience does not supervene on the physical in Chalmers (1996), which was the starting point of (epistemic) two-dimensional semantics.

Building on the discussion in Chalmers (2006, section 3.5), we can formalize the conceivability/possibility link by noting that Chalmers's notion of semantic neutrality can be formalized by calling a formula $\varphi$ neutral in a model $\mathcal{M}$ based on a matrix FWDE if its truth is independent of the epistemic index, i.e., if for any $\langle x, y\rangle$ and $\left\langle x^{\prime}, y^{\prime}\right\rangle$, if $x=x^{\prime}$, then the formula is true in $\langle x, y\rangle$ if and only if it is true in $\left\langle x^{\prime}, y^{\prime}\right\rangle$. It is straightforward to prove that if $\varphi$ is neutral in $\mathcal{M}$, then $C \varphi \leftrightarrow \diamond \varphi$ is true in every point in $\mathcal{M}$, one direction of which we can see as a formal representation of Chalmers's claim that semantically neutral sentences which are conceivable are also possible. This has to be qualified if $C$ does not adequately formalize conceivability, but in any case, we get an apriority/necessity link via $A \varphi \leftrightarrow \square \varphi$, which should do the relevant work. In contrast, there is no obvious way of making sense of the conceivability/possibility link on product matrix FWDEs. In particular, note that the notion of a formula being neutral in a model can straightforwardly be adapted to models based on product matrix FWDEs, but that we cannot prove that if a formula $\varphi$ is 
neutral in a model $\mathcal{M}$ based on a product matrix FWDE, then $C \varphi \leftrightarrow \diamond \varphi$ is true in every point in $\mathcal{M}$. In fact, we can prove that for such a $\varphi$ and $\mathcal{M}, C \varphi \leftrightarrow A \varphi$ is true in every point in $\mathcal{M}$, but the corresponding conceivability/apriority link for semantically neutral sentences is philosophically completely unmotivated.

The notion of a neutral formula is not only useful in bringing out the difference between matrix FWDEs and product matrix FWDEs, but interesting in its own right as a way of formally capturing part of the connection between necessity and apriority in two-dimensional semantics. From this perspective, it is interesting to consider the logic of models based on matrix FWDEs in which every proposition letter is neutral; this is investigated in Fritz (2011, section 4.1.).

Product matrix FWDEs therefore don't constitute a plausible formalization of versions of two-dimensional semantics committed to the conceivability-possibility link. This observation can be used to bring out another interesting point concerning the motivations for two-dimensional semantics: Some philosophers might be attracted to two-dimensional semantics because it provides a compelling way of combining a possible world semantics for apriority with a two-dimensional semantics for indexicals along the lines of Kaplan (1989) which deals well with standard examples of contingent a priori truths involving indexicals. We can use product matrix FWDEs to show that two-dimensional semantics is not the only formal semantics of necessity and apriority which has this feature: product matrix FWDEs provide a possible world semantics for apriority and deal well with standard examples of contingent a priori truths involving indexicals, and although the Kaplanian two-dimensional semantics of indexicals is not immediately recognizable from the definition of product matrix FWDEs, we can define a variant of the model theory in which the connection is more transparent and which is equivalent in all important respects.

Roughly, the idea is the following: Just as we can formulate the standard model theory for the logic of necessity and actuality either using a distinguished world or equivalently using double-indexing (see, e.g., Davies \& Humberstone (1980, pp. 4-5)), so we can formulate a model theory for the logic of necessity, actuality and apriority using doubleindexing and a distinguished world (as in product matrix FWDEs) or equivalently using triple-indexing. Somewhat more formally, we can define our variant of product matrix FWDEs by starting with a set $S$, and letting our set of points of evaluation be the set of triples $S^{3}$. In such a tripel $\langle x, y, z\rangle, x$ represents a metaphysical possibility, $y$ an epistemic possibility, and $z$ a context of utterance. Necessity and apriority are interpreted as before, keeping the context of utterance fixed, whereas actuality now is interpreted as truth in the metaphysical possibility corresponding to the context of utterance, keeping the epistemic possibility and the context of utterance fixed. Formally, the accessibility relations would be given by the following clauses:

- $\langle x, y, z\rangle R_{\square}\left\langle x^{\prime}, y^{\prime}, z^{\prime}\right\rangle$ iff $y=y^{\prime}$ and $z=z^{\prime}$

- $\langle x, y, z\rangle R_{@}\left\langle x^{\prime}, y^{\prime}, z^{\prime}\right\rangle$ iff $y=y^{\prime}$ and $z=z^{\prime}$ and $x^{\prime}=z$

- $\langle x, y, z\rangle R_{A}\left\langle x^{\prime}, y^{\prime}, z^{\prime}\right\rangle$ iff $x=x^{\prime}$ and $z=z^{\prime}$

It is then natural to distinguish the points $\langle x, y, z\rangle$ such that $x=y=z$; it is straightforward to show that the resulting class of FWDEs characterizes $\mathbf{S 5}^{2} @$ as well. Furthermore, we can recover the Kaplanian double-indexing semantics for the actuality operator from this semantics by omitting the second index and the modality $A$. Thus two-dimensional semantics is not the only plausible way of combining a possible world semantics for apriority with a Kaplanian semantics for indexicals. An argument for two-dimensional semantics, assuming that it is correctly formalized by matrix FWDEs, over a semantic theory which is best formalized in terms of product matrix FWDEs would have to appeal to different 
considerations. In particular, it would have to address the nesting problem, and argue for the controversial claim that $N 1$ rather than $N 2$ is informally valid. In fact, as far as the validities in the language of necessity, actuality and apriority is concerned, we have seen that in a certain way, the question which one of $N 1$ and $N 2$ is informally valid encodes all that separates the two theories. This suggests that finding the correct answer to the nesting problem plays an important role in understanding the interactions of necessity and apriority, and so that this problem deserves more attention than it has received so far.

§6. Acknowledgments. This paper is partly based on my master's thesis, written in 2011 at the ILLC, University of Amsterdam, and supervised by Paul Dekker and Yde Venema. I thank them, as well as Andrew Bacon, David Chalmers, James Davies, Jeremy Goodman, Robert Michels, David Ripley, Robert van Rooij, Wolfgang Schwarz and Timothy Williamson for discussions on the material in this paper. I also thank audiences in Amsterdam, Stockholm, Toronto, London, Bochum and Irvine as well as two anonymous reviewers for this journal for comments. This article was written while I was supported by a doctoral studentship from the Arts and Humanities Research Council.

\section{BIBLIOGRAPHY}

Bezhanishvili, N. (2002). Varieties of two-dimensional cylindric algebras. Part I: Diagonalfree case. Algebra Universalis, 48, 11-42.

Bezhanishvili, N., \& Marx, M. (2003). All proper normal extensions of S5-square have the polynomial size property. Studia Logica, 73, 367-382.

Blackburn, P., de Rijke, M., \& Venema, Y. (2001). Modal Logic, Vol. 53 , Cambridge Tracts in Theoretical Computer Science. Cambridge: Cambridge University Press.

Boolos, G. (1993). The Logic of Provability. Cambridge: Cambridge University Press.

Bull, R. A. (1966). That all normal extensions of S4.3 have the finite model property. Zeitschrift für mathematische Logik und Grundlagen der Mathematik, 12, 341-344.

Burgess, J. P. (1999). Which modal logic is the right one? Notre Dame Journal of Formal Logic, 40, 81-93.

Chalmers, D. J. (1996). The Conscious Mind. Oxford: Oxford University Press.

Chalmers, D. J. (2004). Epistemic two-dimensional semantics. Philosophical Studies, 118, 153-226.

Chalmers, D. J. (2006). The foundations of two-dimensional semantics. In GarcíaCarpintero, M., \& Macià, J., editors. Two-Dimensional Semantics, Oxford: Oxford University Press, pp. 55-140.

Chalmers, D. J. (2011). Propositions and attitude ascriptions: A Fregean account. Noûs, 45, 595-639.

Chalmers, D. J., \& Rabern, B. (forthcoming). Two-dimensional semantics and the nesting problem. Analysis.

Cresswell, M. J. (2009). Non-denumerable infinitary modal logic. Journal of Universal Computer Science, 14, 63-71.

Crossley, J. N., \& Humberstone, L. (1977). The logic of "actually". Reports on Mathematical Logic, 8, 11-29.

Davies, M., \& Humberstone, L. (1980). Two notions of necessity. Philosophical Studies, 38, 1-30.

Dugundji, J. (1940). Note on a property of matrices for Lewis and Langford's calculi of propositions. Journal of Symbolic Logic, 5, 150-151. 
Fritz, P. (2011). Matrices and modalities: On the logic of two-dimensional semantics. Master's thesis, Institute for Logic, Language and Computation, University of Amsterdam. Available from: http://www.illc.uva.nl/Research/Reports/MoL-2011-02.text.pdf.

Fritz, P. (2013). A logic for epistemic two-dimensional semantics. Synthese, 190, $1753-1770$.

Gabbay, D. M., Kurucz, A., Wolter, F., \& Zakharyaschev, M. (2003). Many-Dimensional Modal Logics: Theory and Applications, Vol. 148, Studies in Logic and the Foundations of Mathematics. Amsterdam: Elsevier.

Gabbay, D. M., \& Shehtman, V. B. (1998). Products of modal logics, part 1. Logic Journal of the IGPL, 6, 73-146.

Gregory, D. (2011). Iterated modalities, meaning and a priori knowledge. Philosophers' Imprint, 11, 1-11.

Gärdenfors, P. (1973). On the extensions of S5. Notre Dame Journal of Formal Logic, 14, 277-280.

Hanson, W. H. (2006). Actuality, necessity, and logical truth. Philosophical Studies, 130, 437-459.

Hazen, A. (1978). The eliminability of the actuality operator in propositional modal logic. Notre Dame Journal of Formal Logic, 19, 617-622.

Hazen, A. P., Rin, B. G., \& Wehmeier, K. F. (2013). Actuality in propositional modal logic. Studia Logica, 101, 487-503.

Hughes, G. E., \& Cresswell, M. J. (1996). A New Introduction to Modal Logic. London: Routledge.

Humberstone, L. (2004). Two-dimensional adventures. Philosophical Studies, 118, 17-65.

Humberstone, L. (2011). The Connectives. Cambridge, MA: MIT Press.

Kamp, H. (1971). Formal properties of 'now'. Theoria, 37, 227-273.

Kaplan, D. (1989). Demonstratives. In Almog, J., Perry, J., \& Wettstein, H., editors. Themes from Kaplan. Oxford: Oxford University Press, pp. 481-563.

Kreisel, G. (1967). Informal rigour and completeness proofs. In Lakatos, I., editor. Problems in the Philosophy of Mathematics. Amsterdam: North-Holland, pp. 138-186.

Kripke, S. A. (1965). Semantical analysis of modal logic II. Non-normal modal propositional calculi. In Addison, J. W., Henkin, L., \& Tarski, A., editors. The Theory of Models. Amsterdam: North-Holland, pp. 206-220.

Kripke, S. A. (1972). Naming and necessity. In Davidson, D., \& Harman, G., editors. Semantics of Natural Language. Dordrecht: D. Reidel, pp. 253-355, 763-769.

Prior, A. N. (1961). On a family of paradoxes. Notre Dame Journal of Formal Logic, 2, $16-32$.

Prior, A. N. (1968). "Now". Noûs, 2, 101-119.

Restall, G. (2012). A cut-free sequent system for two-dimensional modal logic, and why it matters. Annals of Pure and Applied Logic, 163, 1611-1623.

Salmon, N. (1989). The logic of what might have been. Philosophical Review, 98, 3-34.

Scroggs, S. J. (1951). Extensions of the Lewis system S5. Journal of Symbolic Logic, 16, $112-120$.

Segerberg, K. (1971). An Essay in Classical Modal Logic. vol. 13, Filosofiska Studier. Uppsala: Uppsala Universitet.

Smith, P. (2011). Squeezing arguments. Analysis, 71, 22-30.

Soames, S. (2005). Reference and Description: The Case Against Two-Dimensionalism. Princeton: Princeton University Press.

Tarski, A. (2002). On the concept of following logically. History and Philosophy of Logic, 23, 155-196. Originally published in Polish and German, 1936. 
Tucker, D., \& Thomason, R. H. (2011). Paradoxes of intensionality. Review of Symbolic Logic, 4, 394-411.

Vlach, F. (1973). 'Now' and 'Then': A Formal Study in the Logic of Tense Anaphora. $\mathrm{Ph}$. D. thesis, University of California, Los Angeles.

Williamson, T. (2013). Modal Logic as Metaphysics. Oxford: Oxford University Press.

\section{JESUS COLLEGE}

TURL STREET

OXFORD, OX1 3DW, UK

E-mail: peter.fritz@philosophy.ox.ac.uk 